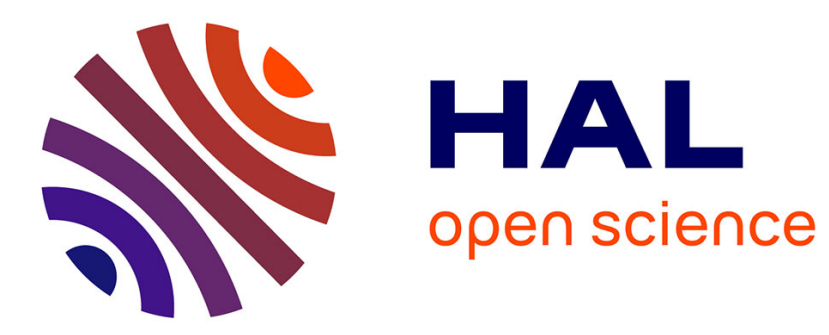

\title{
Some differential systems driven by a fBm with Hurst parameter greater than $1 / 4$
}

Samy Tindel, Iván Torrecilla

\section{To cite this version:}

Samy Tindel, Iván Torrecilla. Some differential systems driven by a fBm with Hurst parameter greater than 1/4. 2009. hal-00352998

\section{HAL Id: hal-00352998 \\ https://hal.science/hal-00352998}

Preprint submitted on 14 Jan 2009

HAL is a multi-disciplinary open access archive for the deposit and dissemination of scientific research documents, whether they are published or not. The documents may come from teaching and research institutions in France or abroad, or from public or private research centers.
L'archive ouverte pluridisciplinaire HAL, est destinée au dépôt et à la diffusion de documents scientifiques de niveau recherche, publiés ou non, émanant des établissements d'enseignement et de recherche français ou étrangers, des laboratoires publics ou privés. 


\title{
SOME DIFFERENTIAL SYSTEMS DRIVEN BY A FBM WITH HURST PARAMETER GREATER THAN 1/4
}

\author{
SAMY TINDEL AND IVÁN TORRECILLA
}

\begin{abstract}
This note is devoted to show how to push forward the algebraic integration setting in order to treat differential systems driven by a noisy input with Hölder regularity greater than $1 / 4$. After recalling how to treat the case of ordinary stochastic differential equations, we mainly focus on the case of delay equations. A careful analysis is then performed in order to show that a fractional Brownian motion with Hurst parameter $H>1 / 4$ fulfills the assumptions of our abstract theorems.
\end{abstract}

\section{INTRODUCTION}

A differential equation driven by a $d$-dimensional fractional Brownian motion $B=$ $\left(B^{1}, \ldots, B^{d}\right)$ is generically written as:

$$
y_{t}=a+\int_{0}^{t} \sigma\left(y_{s}\right) d B_{s}, \quad t \in[0, T]
$$

where $a$ is an initial condition in $\mathbb{R}^{n}, \sigma: \mathbb{R}^{n} \rightarrow \mathbb{R}^{n, d}$ is a smooth enough function, and $T$ is an arbitrary positive constant. The recent developments in rough paths analysis [4, 13, 8] have allowed to solve this kind of differential equation when the Hurst parameter $H$ of the fractional Brownian motion is greater than $1 / 4$, by first giving a natural meaning to the integral $\int_{0}^{t} \sigma\left(y_{s}\right) d B_{s}$ above. It should also be stressed that a great amount of information has been obtained about these systems, ranging from support theorems [7] to the existence of a density for the law of $y_{t}$ at a fixed instant $t$ (see [2, [3]).

In a parallel but somewhat different direction, the algebraic integration theory (introduced in [9]), is meant as an alternative and complementary method of generalized integration with respect to a rough path. It relies on some more elementary and explicit formulae, and its main advantage is that it allows to develop rather easily an intuition about the way to handle differential systems beyond the diffusion case given by (11). This fact is illustrated by the study of delay [16] and Volterra [5] type equations, as well as an attempt to handle partial differential equations driven by a rough path [11]. In each of those cases, the main underlying idea consists in changing slightly the basic structures allowing a generalized integration theory (discrete differential operator $\delta$, sewing map $\Lambda$, controlled processes) in order to adapt them to the context under consideration. While the technical details might be long and tedious, let us insist on the fact that the changes in the structures we have alluded to are always natural and (almost) straightforward. Some twisted Lévy areas also enter into the game in a natural manner.

However, all the results contained in the references mentioned above concern a fractional Brownian motion $B$ with Hurst parameter $H>1 / 3$, while the usual rough path theory enables to handle any $H>1 / 4$ (see [4] for the explicit application to fBm). The current

Date: January 14, 2009.

2000 Mathematics Subject Classification. 60H05, 60H07, 60G15.

Key words and phrases. Rough paths theory; Stochastic delay equations; Fractional Brownian motion. 
paper can then be seen as a step in order to fill this gap, and we shall deal mainly with two kind of systems: first of all, we will show how to solve equation (11) when $1 / 4<H \leq 1 / 3$, thanks to the algebraic integration theory. The results we will obtain are not new, and the algebraic integration formalism has been extended to a much broader context in [10] by means of a tree-based expansion (let us mention again that the case $H>1 / 4$ is also covered by the usual rough path theory). This study is thus included here as a preliminary step, where the changes in the structures (new definition of a controlled path, introduction of a Lévy volume) can be exhibited in a simple enough manner.

Then, in a second part of the paper, we show how to adapt our formalism in order to deal with delay equations of the form:

$$
\left\{\begin{array}{l}
d y_{t}=\sigma\left(y_{t}, y_{t-r_{1}}, \ldots, y_{t-r_{q}}\right) d B_{t} \quad t \in[0, T] \\
y_{t}=\xi_{t}, \quad t \in\left[-r_{q}, 0\right]
\end{array}\right.
$$

where $y$ is a $\mathbb{R}^{n}$-valued continuous process, $q$ is a positive integer, $\sigma: \mathbb{R}^{n, q+1} \rightarrow \mathbb{R}^{n, d}$ is a smooth enough function, $B$ is a $d$-dimensional fractional Brownian motion with Hurst parameter $H>1 / 4$ and $T$ is an arbitrary positive constant. The delay in our equation is represented by the family $0<r_{1}<\ldots<r_{q}<\infty$, and the initial condition $\xi$ is taken as a regular enough deterministic function on $\left[-r_{q}, 0\right]$. Though this kind of system is implicitly considered in [12] in the usual Brownian case, and in [6] for a Hurst parameter $H>1 / 2$, the rough paths techniques have only been used in this context (to the best of our knowledge) in [16], where a delay equation driven by a fractional Brownian motion with Hurst parameter $H>1 / 3$ is considered. Our paper is thus an extension of this last result, and we shall obtain an existence and uniqueness theorem for equation (2) in the case $H>1 / 4$, under reasonable regularity conditions on $\sigma$ and $\xi$.

From our point of view the example of delay equations, which is interesting in its own right because of its potential physical applications, is also worth studying in order to see the kind of algebraical structures which pop out when changing the type of rough differential system we are trying to handle. In case of a delay equation driven by a rough path of order 3 like ours, we shall introduce the notion of doubly delayed controlled processes, and have to assume a priori the existence of some doubly delayed elements of area and volume associated to $B$. This rich structure induces some cumbersome computations when one decides to expand all the calculations explicitly like we did. However, in the end, one also gets the satisfaction to see that the algebraic integration setting is flexible enough to be adapted naturally to many situations. Let us also mention that the infinite dimensional setting of [14] is avoided here, and that all our considerations only involve paths taking values in a finite dimensional space.

Let us also mention that, as in other examples of fractional differential systems, an important part of our work consists in verifying that the fractional Brownian motion satisfies the assumptions of our abstract theorems. The main available tools we are aware of for this kind of task are based on Russo-Vallois approximations [19], analytic approximations of the fBm (like we did in [5]) or Malliavin calculus. We have chosen here to work under this latter framework, since it leads to reasonably short calculations, and also because it allows us to build on the previous results obtained in [16, where this formalism was also adopted.

Here is how our article is structured: Section 2 is devoted to recall the basic ingredients of the algebraic integration setting. The diffusion case is treated at Section B, and the 
bulk of the computations concerning delay systems can be found at Section 4 . Finally, the application to fractional Brownian motion is given at Section 5 .

\section{INCREMENTS}

To begin with, let us present the very basic algebraic structures which will allow to define a pathwise integral with respect to irregular functions.

2.1. Basic notions of algebraic integration. For an arbitrary real number $T>0$, a vector space $V$ and an integer $k \geq 1$ we denote by $\mathcal{C}_{k}(V)$ the set of functions $g:[0, T]^{k} \rightarrow$ $V, g\left(t_{1}, \ldots, t_{k}\right)=g_{t_{1} \ldots t_{k}}$ such that $g_{t_{1} \ldots t_{k}}=0$ whenever $t_{i}=t_{i+1}$ for some $1 \leq i \leq k-1$. Such a function will be called a $(k-1)$-increment, and we will set $\mathcal{C}_{*}(V)=\cup_{k \geq 1} \mathcal{C}_{k}(V)$.

On $\mathcal{C}_{k}(V)$ we introduce the operator $\delta$ defined as follows:

$$
\delta: \mathcal{C}_{k}(V) \rightarrow \mathcal{C}_{k+1}(V), \quad(\delta g)_{t_{1} \cdots t_{k+1}}=\sum_{i=1}^{k+1}(-1)^{k-i} g_{t_{1} \cdots \hat{t}_{i} \cdots t_{k+1}}
$$

where $\hat{t}_{i}$ means that this particular argument is omitted. A fundamental property of $\delta$, which is easily verified, is that $\delta \circ \delta=0$. We will denote $\mathcal{Z C}_{k}(V)=\mathcal{C}_{k}(V) \cap \operatorname{Ker} \delta$ and $\mathcal{B C}_{k}(V)=\mathcal{C}_{k}(V) \cap \operatorname{Im} \delta$.

Throughout the paper we will mainly deal with actions of $\delta$ on $\mathcal{C}_{i}, i=1,2$. That is, consider $g \in \mathcal{C}_{1}$ and $h \in \mathcal{C}_{2}$. Then, for any $s, u, t \in[0, T]$, we have

$$
(\delta g)_{s t}=g_{t}-g_{s}, \quad \text { and } \quad(\delta h)_{s u t}=h_{s t}-h_{s u}-h_{u t} .
$$

Furthermore, it is easily checked that $\mathcal{Z C}_{k+1}(V)=\mathcal{B C}_{k}(V)$ for any $k \geq 1$. In particular, we have the following property:

Lemma 2.1. Let $k \geq 1$ and $h \in \mathcal{Z C}_{k+1}(V)$. There exists a (non unique) $f \in \mathcal{C}_{k}(V)$ such that $h=\delta f$.

Lemma 2.1 implies that all the elements $h \in \mathcal{C}_{2}(V)$ such that $\delta h=0$ can be written as $h=\delta f$ for some (non unique) $f \in \mathcal{C}_{1}(V)$. Thus we have a heuristic interpretation of $\left.\delta\right|_{\mathcal{C}_{2}(V)}$ as a measure of how much a given 1-increment is far from being an exact increment of a function, i.e., a finite difference.

Remark 2.1. Here is a first elementary but important link between these algebraic structures and integration theory. Let $f$ and $g$ be two smooth real valued functions on $[0, T]$. Define $I \in \mathcal{C}_{2}$ by

$$
I_{s t}=\int_{s}^{t}\left(\int_{s}^{v} d g_{w}\right) d f_{v}, \quad \text { for } \quad s, t \in[0, T] .
$$

Then, $(\delta I)_{s u t}=\left[g_{u}-g_{s}\right]\left[f_{t}-f_{u}\right]=(\delta g)_{s u}(\delta f)_{u t}$. Hence we see that the operator $\delta$ transforms iterated integrals into products of increments, and we will be able to take advantage of both regularities of $f$ and $g$ in these products of the form $\delta g \delta f$.

Let us concentrate now on the case $V=\mathbb{R}^{d}$, and notice that our future discussions will mainly rely on $k$-increments with $k \leq 2$, for which we will use some analytical assumptions. Namely, we measure the size of these increments by Hölder-type norms defined in the following way. For $f \in \mathcal{C}_{2}(V)$ and $\mu \in(0, \infty)$, let

$$
\|f\|_{\mu}=\sup _{s, t \in[0, T]} \frac{\left|f_{s t}\right|}{|t-s|^{\mu}}
$$


and set $\mathcal{C}_{2}^{\mu}(V)=\left\{f \in \mathcal{C}_{2}(V) ;\|f\|_{\mu}<\infty\right\}$.

The usual Hölder spaces $\mathcal{C}_{1}^{\mu}(V)$ will be determined in the following way. For a continuous function $g \in \mathcal{C}_{1}(V)$, we simply set

$$
\|g\|_{\mu}=\|\delta g\|_{\mu}
$$

where the right-hand side of this equality is defined after (5); we will say that $g \in \mathcal{C}_{1}^{\mu}(V)$ iff $\|g\|_{\mu}$ is finite. Notice that $\|\cdot\|_{\mu}$ is only a semi-norm on $\mathcal{C}_{1}(V)$. However we will generally work on spaces of the type

$$
\mathcal{C}_{1, a}^{\mu}(V)=\left\{g:[0, T] \rightarrow V ; g_{0}=a,\|g\|_{\mu}<\infty\right\},
$$

for a given $a \in V$, on which $\|g\|_{\mu}$ then becomes a norm. For $h \in \mathcal{C}_{3}(V)$ we set

$$
\begin{aligned}
\|h\|_{\gamma, \rho} & =\sup _{s, u, t \in[0, T]} \frac{\left|h_{\text {sut }}\right|}{|u-s|^{\gamma}|t-u|^{\rho}} \\
\|h\|_{\mu} & =\inf \left\{\sum_{i}\left\|h_{i}\right\|_{\rho_{i}, \mu-\rho_{i}} ; h=\sum_{i} h_{i}, 0<\rho_{i}<\mu\right\},
\end{aligned}
$$

where the last infimum is taken over all sequences $\left\{h_{i} \in \mathcal{C}_{3}(V)\right\}$ such that $h=\sum_{i} h_{i}$. Then $\|\cdot\|_{\mu}$ is easily seen to be a norm on $\mathcal{C}_{3}(V)$, and we set

$$
\mathcal{C}_{3}^{\mu}(V):=\left\{h \in \mathcal{C}_{3}(V) ;\|h\|_{\mu}<\infty\right\} .
$$

Eventually, let $\mathcal{C}_{j}^{1+}(V)=\cup_{\mu>1} \mathcal{C}_{j}^{\mu}(V), j=1,2,3$, and remark that the same kind of norms can be considered on the spaces $\mathcal{Z C}_{3}(V)$, leading to the definition of some spaces $\mathcal{Z C}_{3}^{\mu}(V)$ and $\mathcal{Z C}_{3}^{1+}(V)$.

With these notations in mind, the crucial point in our approach to pathwise integration of irregular processes is that, under mild smoothness conditions, the operator $\delta$ can be inverted. This inverse is called $\Lambda$, and is defined in the following proposition, whose proof can be found in [9, 11:

Proposition 2.2. There exists a unique linear map $\Lambda: \mathcal{Z C}_{3}^{1+}(V) \rightarrow \mathcal{C}_{2}^{1+}(V)$ such that

$$
\delta \Lambda=I d_{\mathcal{Z C}_{3}^{1+}(V)} \quad \text { and } \quad \Lambda \delta=I d_{\mathcal{C}_{2}^{1+}(V)} .
$$

In other words, for any $h \in \mathcal{C}_{3}^{1+}(V)$ such that $\delta h=0$ there exists a unique $g=\Lambda(h) \in$ $\mathcal{C}_{2}^{1+}(V)$ such that $\delta g=h$. Furthermore, for any $\mu>1$, the map $\Lambda$ is continuous from $\mathcal{Z C}_{3}^{\mu}(V)$ to $\mathcal{C}_{2}^{\mu}(V)$ and we have

$$
\|\Lambda h\|_{\mu} \leq \frac{1}{2^{\mu}-2}\|h\|_{\mu}, \quad h \in \mathcal{Z} \mathcal{C}_{3}^{\mu}(V) .
$$

It is worth mentioning at this point that $\Lambda$ gives raise to a kind of generalized Young integral, which is a second link between the algebraic structures introduced so far and a theory of generalized integration:

Corollary 2.3. For any 1-increment $g \in \mathcal{C}_{2}(V)$ such that $\delta g \in \mathcal{C}_{3}^{1+}$,

$$
(I d-\Lambda \delta) g=\lim _{\left|\Pi_{s t}\right| \rightarrow 0} \sum_{i=0}^{n} g_{t_{i} t_{i+1}},
$$

where the limit is over any partition $\Pi_{s t}=\left\{t_{0}=s, \ldots, t_{n}=t\right\}$ of $[s, t]$, whose mesh tends to zero. Thus by setting $\delta f=(I d-\Lambda \delta) g$, the 1-increment $\delta f$ is the indefinite integral of the 1-increment $g$. 
We can now explain heuristically how our generalized integral will be defined.

Remark 2.2. Let $f$ and $g$ be two real valued smooth functions, and define $I \in \mathcal{C}_{2}$ like in Remark 2.1. Thanks to this remark and Proposition 2.2, the following decompositionrecomposition for $I=\int d f \int d g$ holds true:

$$
\int d g \int d f \stackrel{\delta}{\longrightarrow}(\delta g)(\delta f) \stackrel{\Lambda}{\longrightarrow} \int d g \int d f,
$$

where for the second step of this construction, we have only used the fact that the product of increments $(\delta g)(\delta f)$, considered as an element of $\mathcal{Z C}_{3}$, is smooth enough. This simple procedure allows then to extend the notion of iterated integral to a non-smooth situation, by just applying the operator $\Lambda$ to $(\delta g)(\delta f)$ whenever we are allowed to do it.

2.2. Some further notations. We summarize in this section some of the notation which will be used throughout the paper.

A multilinear operator $A$ of order $l$, from $\mathbb{R}^{d_{1}} \times \ldots \times \mathbb{R}^{d_{l}}$ to $\mathbb{R}^{n}$, is denoted as an element $A \in \mathbb{R}^{n, d_{1}, \ldots, d_{l}}$. In order to avoid tricky matrix notations, we have decided to expand all our computations in coordinates, and use Einstein's convention on summations over repeated indices. Notice that we will also use the notation $A \in \mathbb{R}^{d_{1}, d_{2}, d_{3}, d_{4}}$ for a linear operator from $\mathbb{R}^{d_{3}, d_{4}}$ to $\mathbb{R}^{d_{1}, d_{2}}$. We hope that this convention won't lead to any ambiguity. The transposed of a matrix $M \in \mathbb{R}^{d_{1}, d_{2}}$ is written as $M^{*}$.

For a function $\varphi:\left(\mathbb{R}^{n}\right)^{q+1} \rightarrow \mathbb{R}$, we denote by $\partial_{i}^{j} \varphi\left(w_{0}, w_{1}, \ldots, w_{q}\right)$ the derivative of $\varphi$ with respect to the $i^{\text {th }}$ component of $w_{j}$, for $i \leq n$ and $j=0, \ldots, q$.

We shall meet two kind of products of increments: first, for $g \in \mathcal{C}_{n}\left(\mathbb{R}^{l, d}\right)$ and $h \in \mathcal{C}_{m}\left(\mathbb{R}^{d}\right)$ we set $g h$ for the element of $\mathcal{C}_{n+m-1}\left(\mathbb{R}^{l}\right)$ defined by

$$
(g h)_{t_{1}, \ldots, t_{m+n-1}}=g_{t_{1}, \ldots, t_{n}} h_{t_{n}, \ldots, t_{m+n-1}}, \quad t_{1}, \ldots, t_{m+n-1} \in[0, T] .
$$

If now $g \in \mathcal{C}_{n}\left(\mathbb{R}^{l, d}\right)$ and $h \in \mathcal{C}_{n}\left(\mathbb{R}^{d}\right)$ we set $g \cdot h$ for the element of $\mathcal{C}_{n}\left(\mathbb{R}^{l}\right)$ defined by

$$
(g \cdot h)_{t_{1}, \ldots, t_{n}}=g_{t_{1}, \ldots, t_{n}} h_{t_{1}, \ldots, t_{n}}, \quad t_{1}, \ldots, t_{n} \in[0, T] .
$$

In order to avoid ambiguities, we shall denote by $\mathcal{N}\left[f ; \mathcal{C}_{j}^{\kappa}\right]$ the $\kappa$-Hölder norm on the space $\mathcal{C}_{j}$, for $j=1,2,3$. For $\zeta \in \mathcal{C}_{1}(V)$, we also set $\mathcal{N}\left[\zeta ; \mathcal{C}_{1}^{\infty}(V)\right]=\sup _{0 \leq s \leq T}\left|\zeta^{i}\right|_{V}$.

The integral of a real valued function $f$ with respect to another real valued function $g$, when properly defined, is written indistinctly as $\int f d g$ or $\mathcal{J}(f d g)$.

\section{THE DIFFUSION CASE}

In this section, we will recall the basic steps which allow to define rigorously and solve an equation of the form:

$$
y_{t}=a+\int_{0}^{t} \sigma\left(y_{s}\right) d x_{s}, \quad t \in[0, T]
$$

where $a$ is an initial condition in $\mathbb{R}^{n}, \sigma: \mathbb{R}^{n} \rightarrow \mathbb{R}^{n, d}$ is a smooth enough function, $T$ is an arbitrary positive constant, and $x$ is a generic $d$-dimensional noisy input with Hölder regularity $\gamma>1 / 4$. In the algebraic integration setting [9, 10], this task amounts to perform the following steps:

(1) Definition of an incremental operator $\delta$ and its inverse $\Lambda$.

(2) Definition of a suitable notion of controlled processes, and integration of those processes with respect to $x$. 
(3) Resolution of the equation thanks to a fixed point procedure in the space of controlled processes.

Having dealt with the first of those points at Section 2.1, we turn now to the second one, that is a definition of a useful notion of controlled processes.

3.1. Weakly controlled processes. Before giving the formal definition of a weakly controlled process in the context of equation (9), let us recall that when the regularity of the noise is $\gamma>1 / 4$, the rough path setting relies on the a priori existence of an area (resp. volume) element $\mathbf{x}^{2}$ (resp. $\mathbf{x}^{\mathbf{3}}$ ) satisfying the so-called Chen's relations:

Hypothesis 3.1. The path $\mathbb{R}^{d}$-valued $x$ is $\gamma$-Hölder continuous with $\gamma>1 / 4$, and admits a Lévy area and a volume element, that is two increments $\mathbf{x}^{2} \in \mathcal{C}_{2}^{2 \gamma}\left(\mathbb{R}^{d, d}\right)$ and $\mathbf{x}^{\mathbf{3}} \in$ $\mathcal{C}_{2}^{3 \gamma}\left(\mathbb{R}^{d, d, d}\right)$ (which represent respectively $\mathcal{J}(d x d x)$ and $\mathcal{J}(d x d x d x)$, with the conventions of Section 2.2) satisfying:

$$
\begin{aligned}
& \delta \mathbf{x}^{2}=\delta x \otimes \delta x, \quad \text { i.e. }\left(\delta\left(\mathbf{x}^{2}\right)^{i j}\right)_{s u t}=\left(\delta x^{i}\right)_{s u}\left(\delta x^{j}\right)_{u t} \\
& \delta \mathbf{x}^{3}=\mathbf{x}^{\mathbf{2}} \otimes \delta x+\delta x \otimes \mathbf{x}^{\mathbf{2}}, \quad \text { i.e. }\left(\delta\left(\mathbf{x}^{\mathbf{3}}\right)^{i j k}\right)_{s u t}=\left(\mathbf{x}_{s u}^{2}\right)^{i j}\left(\delta x^{k}\right)_{u t}+\left(\delta x^{i}\right)_{s u}\left(\mathbf{x}_{u t}^{2}\right)^{j k}
\end{aligned}
$$

for any $s, u, t \in[0, T]$, and any $i, j, k \in\{1, \ldots, d\}$.

The geometrical assumption for rough paths (which is satisfied by the fractional Brownian motion in the Stratonovich setting) also states that products of increments should be expressed in terms of iterated integrals:

Hypothesis 3.2. Let $\mathrm{x}^{2}$ be the area process defined at Hypothesis 3.1, and denote by $\mathbf{x}^{2, \mathbf{s}}$ the symmetric part of $\mathbf{x}^{2}$, i.e. $\mathbf{x}^{2, \mathbf{s}}=\frac{1}{2}\left(\mathbf{x}^{2}+\left(\mathbf{x}^{2}\right)^{*}\right)$. Then we suppose that for $0 \leq s<t \leq T$ we have:

$$
\mathbf{x}_{s t}^{\mathbf{2}, \mathbf{s}}=\frac{1}{2}(\delta x)_{s t} \otimes(\delta x)_{s t} .
$$

With these hypotheses in mind, the natural class of processes which will be integrated against $x$ are processes whose increments can be expressed simply enough in terms of the increments of $x$ :

Definition 3.1. Let $z$ be a process in $\mathcal{C}_{1}^{\kappa}\left(\mathbb{R}^{l}\right)$ with $\kappa \leq \gamma$ and $3 \kappa+\gamma>1$, such that $z_{0}=a \in \mathbb{R}^{l}$. We say that $z$ is a weakly controlled path based on $x$ if $\delta z \in \mathcal{C}_{2}^{\kappa}\left(\mathbb{R}^{l}\right)$ can be decomposed into

$$
\delta z^{i}=\left(\zeta^{1}\right)^{i j} \delta x^{j}+\left(\zeta^{2}\right)^{i j k}\left(\mathbf{x}^{2}\right)^{k j}+r^{i}, \quad \text { i.e. } \quad\left(\delta z^{i}\right)_{s t}=\left(\zeta_{s}^{1}\right)^{i j}\left(\delta x^{j}\right)_{s t}+\left(\zeta_{s}^{2}\right)^{i j k}\left(\mathbf{x}_{s t}^{\mathbf{2}}\right)^{k j}+r_{s t}^{i},
$$

for any $1 \leq i \leq l, 1 \leq j, k \leq d$. In the previous decomposition, we further assume that $\zeta^{1} \in \mathcal{C}_{1}^{\kappa}\left(\mathbb{R}^{l, d}\right)$ is a path with a given initial condition $\zeta_{0}^{1}=b \in \mathbb{R}^{l, d}$, such that $\delta \zeta^{1} \in \mathcal{C}_{2}^{\kappa}\left(\mathbb{R}^{l, d}\right)$ can be decomposed itself into:

$$
\delta\left(\zeta^{1}\right)^{i j}=\left(\zeta^{2}\right)^{i j k} \delta x^{k}+\rho^{i j} \text {, i.e. }\left(\delta\left(\zeta^{1}\right)^{i j}\right)_{s t}=\left(\zeta_{s}^{2}\right)^{i j k}\left(\delta x^{k}\right)_{s t}+\rho_{s t}^{i j},
$$

for all $s, t \in[0, T]$, where $\zeta^{2}$ is a given path in $\mathcal{C}_{1}^{\kappa}\left(\mathbb{R}^{l, d, d}\right)$. Notice also that in the previous equations, $r$ and $\rho$ are understood as regular remainders, such that $r \in \mathcal{C}_{2}^{3 \kappa}\left(\mathbb{R}^{l}\right)$ and $\rho \in \mathcal{C}_{2}^{2 \kappa}\left(\mathbb{R}^{l, d}\right)$.

The space of weakly controlled paths will be denoted by $\mathcal{Q}_{\kappa, a, b}\left(\mathbb{R}^{l}\right)$, and a process $z \in$ $\mathcal{Q}_{\kappa, a, b}\left(\mathbb{R}^{l}\right)$ can be considered in fact as a triple $\left(z, \zeta^{1}, \zeta^{2}\right)$. The natural semi-norm on 
$\mathcal{Q}_{\kappa, a, b}\left(\mathbb{R}^{l}\right)$ is given by

$$
\begin{aligned}
\mathcal{N}\left[z ; \mathcal{Q}_{\kappa, a, b}\left(\mathbb{R}^{l}\right)\right] & =\mathcal{N}\left[z ; \mathcal{C}_{1}^{\kappa}\left(\mathbb{R}^{l}\right)\right]+\mathcal{N}\left[\zeta^{1} ; \mathcal{C}_{1}^{\infty}\left(\mathbb{R}^{l, d}\right)\right]+\mathcal{N}\left[\zeta^{1} ; \mathcal{C}_{1}^{\kappa}\left(\mathbb{R}^{l, d}\right)\right] \\
& +\mathcal{N}\left[\zeta^{2} ; \mathcal{C}_{1}^{\infty}\left(\mathbb{R}^{l, d, d}\right)\right]+\mathcal{N}\left[\zeta^{2} ; \mathcal{C}_{1}^{\kappa}\left(\mathbb{R}^{l, d, d}\right)\right] \\
& +\mathcal{N}\left[\rho ; \mathcal{C}_{2}^{2 \kappa}\left(\mathbb{R}^{l, d}\right)\right]+\mathcal{N}\left[r ; \mathcal{C}_{2}^{3 \kappa}\left(\mathbb{R}^{l}\right)\right]
\end{aligned}
$$

where the notations $\mathcal{N}\left[g ; \mathcal{C}_{1}^{\kappa}(V)\right]$ and $\mathcal{N}\left[\zeta ; \mathcal{C}_{1}^{\infty}(V)\right]$ have been introduced at Section 2.9.

Remark 3.1. With respect to the case $\gamma>1 / 3$, the link between $\zeta^{1}$ and $\zeta^{2}$ in the definition of controlled processes is new. This cascade relation between $z, \zeta^{1}$ and $\zeta^{2}$ is reminiscent of the Heinsenberg group structure of Lyons' theory, and is really natural for computational purposes.

We can now study the stability of controlled processes by composition with a regular function.

3.2. Composition of controlled processes. The results of this section can be summarized into the following:

Proposition 3.1. Assume Hypothesis 3.9 holds true. Let $z \in \mathcal{Q}_{\kappa, a, b}\left(\mathbb{R}^{l}\right)$ with decomposition (10), consider a regular function $\varphi \in \mathcal{C}_{b}^{3}\left(\mathbb{R}^{l} ; \mathbb{R}\right)$ and set $\hat{z}=\varphi(z), \hat{a}=\varphi(a)$, $\hat{b}=\partial_{i} \varphi(a) b^{i}$. Then $\hat{z} \in \mathcal{Q}_{\kappa, \hat{a}, \hat{b}}(\mathbb{R})$, and this latter path admits the decomposition

$$
\delta \hat{z}=\left(\hat{\zeta}^{1}\right)^{j} \delta x^{j}+\left(\hat{\zeta}^{2}\right)^{j k}\left(\mathbf{x}^{2}\right)^{k j}+\hat{r}
$$

with

$$
\left(\hat{\zeta}^{1}\right)^{j}=\left[\partial_{i} \varphi(z) \cdot\left(\zeta^{1}\right)^{i j}\right], \quad\left(\hat{\zeta}^{2}\right)^{j k}=\left[\partial_{i} \varphi(z) \cdot\left(\zeta^{2}\right)^{i j k}\right]+\left[\partial_{i_{1} i_{2}} \varphi(z) \cdot\left(\zeta^{1}\right)^{i_{1} j} \cdot\left(\zeta^{1}\right)^{i_{2} k}\right],
$$

and where $\hat{r}$ can be further decomposed into $\hat{r}=\hat{r}^{1}+\hat{r}^{2}+\hat{r}^{3}$, with:

$$
\begin{aligned}
\hat{r}^{1}= & \partial_{i} \varphi(z) r^{i}, \\
\hat{r}^{2}= & \frac{1}{2}\left[\partial_{i_{1} i_{2}} \varphi(z) \cdot\left(\zeta^{2}\right)^{i_{1} j_{1} k_{1}} \cdot\left(\zeta^{2}\right)^{i_{2} j_{2} k_{2}}\right]\left[\left(\mathbf{x}^{2}\right)^{k_{1} j_{1}} \cdot\left(\mathbf{x}^{2}\right)^{k_{2} j_{2}}\right]+\frac{1}{2} \partial_{i_{1} i_{2}} \varphi(z)\left[r^{i_{1}} \cdot r^{i_{2}}\right] \\
& +\left[\partial_{i_{1} i_{2}} \varphi(z) \cdot\left(\zeta^{1}\right)^{i_{1} j_{1}} \cdot\left(\zeta^{2}\right)^{i_{2} j k}\right]\left[\delta x^{j_{1}} \cdot\left(\mathbf{x}^{2}\right)^{k j}\right] \\
& +\left[\partial_{i_{1} i_{2}} \varphi(z) \cdot\left(\zeta^{1}\right)^{i_{1} j}\right]\left[\delta x^{j} \cdot r^{i_{2}}\right]+\left[\partial_{i_{1} i_{2}} \varphi(z) \cdot\left(\zeta^{2}\right)^{i_{1} j k}\right]\left[\left(\mathbf{x}^{2}\right)^{k j} \cdot r^{i_{2}}\right], \\
\hat{r}^{3}= & \delta \varphi(z)-\partial_{i} \varphi(z) \delta z^{i}-\frac{1}{2} \partial_{i j} \varphi(z)\left[\delta z^{i} \cdot \delta z^{j}\right] .
\end{aligned}
$$

As far as $\left(\hat{\zeta}^{1}\right)^{j}$ is concerned, for $1 \leq j \leq d$, it can be decomposed into

$$
\delta\left(\hat{\zeta}^{1}\right)^{j}=\left(\hat{\zeta}^{2}\right)^{j k} \delta x^{k}+\hat{\rho}^{j}
$$

where the remainder $\hat{\rho}^{j}$ can be expressed as $\hat{\rho}^{j}=\left(\hat{\rho}^{1}\right)^{j}+\left(\hat{\rho}^{2}\right)^{j}$, with:

$$
\begin{aligned}
\left(\hat{\rho}^{1}\right)^{j}= & \partial_{i} \varphi(z) \rho^{i j}+\left[\delta\left[\partial_{i} \varphi(z)\right] \cdot \delta\left(\zeta^{1}\right)^{i j}\right] \\
& +\left[\partial_{i_{1} i_{2}} \varphi(z) \cdot\left(\zeta^{1}\right)^{i_{1} j} \cdot\left(\zeta^{2}\right)^{i_{2} j_{2} k_{2}}\right]\left(\mathbf{x}^{2}\right)^{k_{2} j_{2}}+\left[\partial_{i_{1} i_{2}} \varphi(z) \cdot\left(\zeta^{1}\right)^{i_{1} j}\right] r^{i_{2}}, \\
\left(\hat{\rho}^{2}\right)^{j}= & \left(\zeta^{1}\right)^{i_{1} j} \delta\left[\partial_{i_{1}} \varphi(z)\right]-\left[\left(\zeta^{1}\right)^{i_{1} j} \cdot \partial_{i_{1} i_{2}} \varphi(z)\right] \delta z^{i_{2}} .
\end{aligned}
$$

Finally, the following cubical bound holds true for the norm of $\hat{z}$ :

$$
\mathcal{N}\left[\hat{z} ; \mathcal{Q}_{\kappa, \hat{a}, \hat{b}}(\mathbb{R})\right] \leq c_{\varphi, x, T}\left(1+\mathcal{N}^{3}\left[z ; \mathcal{Q}_{\kappa, a, b}\left(\mathbb{R}^{l}\right)\right]\right) .
$$


Proof. This proof is a matter of long and tedious Taylor expansions, and we shall omit most of the details. Let us just mention that we start from the relation:

$$
\begin{aligned}
(\delta \hat{z})_{s t}=\varphi\left(z_{t}\right)-\varphi\left(z_{s}\right)= & \partial_{i} \varphi\left(z_{s}\right)\left(\delta z^{i}\right)_{s t}+\frac{1}{2} \partial_{i_{1} i_{2}} \varphi\left(z_{s}\right)\left(\delta z^{i_{1}}\right)_{s t}\left(\delta z^{i_{2}}\right)_{s t} \\
& +\varphi\left(z_{t}\right)-\varphi\left(z_{s}\right)-\partial_{i} \varphi\left(z_{s}\right)\left(\delta z^{i}\right)_{s t}-\frac{1}{2} \partial_{i_{1} i_{2}} \varphi\left(z_{s}\right)\left(\delta z^{i_{1}}\right)_{s t}\left(\delta z^{i_{2}}\right)_{s t} .
\end{aligned}
$$

The desired decomposition (11) is then obtained by plugging relation (10) into the last identity, and expanding further. It should also be noticed that some cancellations occur due to Hypothesis 3.2. Relation (12) is obtained in the same manner, and our bound (13) is a matter of standard computations once the expressions (11) and (12) are known.

3.3. Integration of controlled paths. It is of course of fundamental importance for our purposes to be able to integrate a controlled process with respect to the driving signal $x$. This is achieved in the following proposition:

Proposition 3.2. For a given $\gamma>1 / 4$ and $\kappa \leq \gamma$, let $x$ be a process satisfying Hypothesis 3.1. Let also $m \in \mathcal{Q}_{\kappa, b, c}\left(\mathbb{R}^{1, d}\right)$ with decomposition $m_{0}=b \in \mathbb{R}^{1, d}$ and

$$
\left(\delta m^{i}\right)_{s t}=\left(\mu_{s}^{1}\right)^{i j}\left(\delta x^{j}\right)_{s t}+\left(\mu_{s}^{2}\right)^{i j k}\left(\mathbf{x}_{s t}^{\mathbf{2}}\right)^{k j}+r_{s t}^{i}, \quad 1 \leq i \leq d,
$$

where $\mu^{1} \in \mathcal{C}_{1}^{\kappa}\left(\mathbb{R}^{d, d}\right), \mu_{0}^{1}=c \in \mathbb{R}^{d, d}$, and where $\delta \mu^{1} \in \mathcal{C}_{2}^{\kappa}\left(\mathbb{R}^{d, d}\right)$ can be decomposed into

$$
\left(\delta\left(\mu^{1}\right)^{i j}\right)_{s t}=\left(\mu_{s}^{2}\right)^{i j k}\left(\delta x^{k}\right)_{s t}+\rho_{s t}^{i j},
$$

with $\mu^{2} \in \mathcal{C}_{1}^{\kappa}\left(\mathbb{R}^{d, d, d}\right), \rho \in \mathcal{C}_{2}^{2 \kappa}\left(\mathbb{R}^{d, d}\right), r \in \mathcal{C}_{2}^{3 \kappa}\left(\mathbb{R}^{1, d}\right)$. Define then $z$ by $z_{0}=a \in \mathbb{R}$ and

$$
\delta z=m^{i} \delta x^{i}+\left(\mu^{1}\right)^{i j}\left(\mathbf{x}^{2}\right)^{j i}+\left(\mu^{2}\right)^{i j k}\left(\mathbf{x}^{\mathbf{3}}\right)^{k j i}+\Lambda\left(r^{i} \delta x^{i}+\rho^{i j}\left(\mathbf{x}^{\mathbf{2}}\right)^{j i}+\delta\left(\mu^{2}\right)^{i j k}\left(\mathbf{x}^{\mathbf{3}}\right)^{k j i}\right) .
$$

Finally, set

$$
\mathcal{J}\left(m^{i} d x^{i}\right)=\delta z
$$

Then,

(i) z is well-defined as an element of $\mathcal{Q}_{\kappa, a, b}(\mathbb{R})$, and $\mathcal{J}\left(m^{i} d x^{i}\right)$ coincides with a Riemann integral in case of some smooth processes $m$ and $x$.

(ii) The semi-norm of $z$ in $\mathcal{Q}_{\kappa, a, b}(\mathbb{R})$ can be estimated as

$$
\mathcal{N}\left[z ; \mathcal{Q}_{\kappa, a, b}(\mathbb{R})\right] \leq c_{x, T}\left\{1+|b|_{\mathbb{R}^{1, d}}+T^{\gamma-\kappa}\left(|b|_{\mathbb{R}^{1, d}}+\mathcal{N}\left[m ; \mathcal{Q}_{\kappa, b, c}\left(\mathbb{R}^{1, d}\right)\right]\right)\right\} .
$$

Furthermore, we obtain

$$
\|\delta z\|_{\kappa} \leq c_{T, x} T^{\gamma-\kappa}\left(|b|_{\mathbb{R}^{1, d}}+\mathcal{N}\left[m ; \mathcal{Q}_{\kappa, b, c}\left(\mathbb{R}^{1, d}\right)\right]\right) .
$$

(iii) It holds

$$
\begin{aligned}
& \mathcal{J}_{s t}\left(m^{i} d x^{i}\right) \\
& =\lim _{\left|\Pi_{s t} \rightarrow 0\right|} \sum_{q=0}^{n}\left[m_{t_{q}}^{i}\left(\delta x^{i}\right)_{t_{q}, t_{q+1}}+\left(\mu_{t_{q}}^{1}\right)^{i j}\left(\mathbf{x}_{t_{q}, t_{q+1}}^{\mathbf{2}}\right)^{j i}+\left(\mu_{t_{q}}^{2}\right)^{i j k}\left(\mathbf{x}_{t_{q}, t_{q+1}}^{\mathbf{3}}\right)^{k j i}\right]
\end{aligned}
$$

for any $0 \leq s<t \leq T$, where the limit is taken over all partitions $\Pi_{s t}=\left\{t_{0}=\right.$ $\left.s, \ldots, t_{n}=t\right\}$ of $[s, t]$, as the mesh of the partition goes to zero. 
Proof. Here again, the proof is long and cumbersome, and we prefer to avoid most of the technical details for sake of conciseness. Let us just try to justify the second part of the first assertion (about Riemann integrals).

Let us suppose then that $x$ is a smooth function and that $m \in \mathcal{C}_{1}^{\infty}\left(\mathbb{R}^{1, d}\right)$ admits the decomposition (14) with $\mu^{1} \in \mathcal{C}_{1}^{\infty}\left(\mathbb{R}^{d, d}\right), \mu^{2} \in \mathcal{C}_{1}^{\infty}\left(\mathbb{R}^{d, d, d}\right), \rho \in \mathcal{C}_{2}^{\infty}\left(\mathbb{R}^{d, d}\right)$ and $r \in \mathcal{C}_{2}^{\infty}\left(\mathbb{R}^{1, d}\right)$. Then $\mathcal{J}\left(m^{i} d x^{i}\right)$ is well-defined, and we have

$$
\int_{s}^{t} m_{u}^{i} d x_{u}^{i}=m_{s}^{i}\left[x_{t}^{i}-x_{s}^{i}\right]+\int_{s}^{t}\left[m_{u}^{i}-m_{s}^{i}\right] d x_{u}^{i}
$$

for $s<t$, which can also be read as:

$$
\mathcal{J}\left(m^{i} d x^{i}\right)=m^{i} \delta x^{i}+\mathcal{J}\left(\delta m^{i} d x^{i}\right) .
$$

Let us now plug the decomposition (14) into the expression (21). This yields

$$
\begin{aligned}
\mathcal{J}\left(m^{i} d x^{i}\right) & =m^{i} \delta x^{i}+\mathcal{J}\left(\left[\left(\mu^{1}\right)^{i j} \delta x^{j}\right] d x^{i}\right)+\mathcal{J}\left(\left[\left(\mu^{2}\right)^{i j k}\left(\mathbf{x}^{\mathbf{2}}\right)^{k j}\right] d x^{i}\right)+\mathcal{J}\left(r^{i} d x^{i}\right) \\
& =m^{i} \delta x^{i}+\left(\mu^{1}\right)^{i j}\left(\mathbf{x}^{\mathbf{2}}\right)^{j i}+\left(\mu^{2}\right)^{i j k}\left(\mathbf{x}^{\mathbf{3}}\right)^{k j i}+\mathcal{J}\left(r^{i} d x^{i}\right),
\end{aligned}
$$

and observe that the terms $m^{i} \delta x^{i},\left(\mu^{1}\right)^{i j}\left(\mathbf{x}^{2}\right)^{j i}$ and $\left(\mu^{2}\right)^{i j k}\left(\mathbf{x}^{3}\right)^{k j i}$ in (22) are well-defined provided that $x, \mathbf{x}^{2}$ and $\mathbf{x}^{3}$ are defined themselves. To push forward our analysis to the rough case, we still need to handle the term $\mathcal{J}\left(r^{i} d x^{i}\right)$. Owing to (22) we can write

$$
\mathcal{J}\left(r^{i} d x^{i}\right)=\mathcal{J}\left(m^{i} d x^{i}\right)-m^{i} \delta x^{i}-\left(\mu^{1}\right)^{i j}\left(\mathbf{x}^{\mathbf{2}}\right)^{j i}-\left(\mu^{2}\right)^{i j k}\left(\mathbf{x}^{\mathbf{3}}\right)^{k j i},
$$

and let us analyze this relation by applying $\delta$ to both sides of the last identity. Invoking standard rules on the operator $\delta$, and the fact that $x$ satisfies Hypothesis 3.1. we end up with:

$$
\delta\left[\mathcal{J}\left(r^{i} d x^{i}\right)\right]=\delta\left(\mu^{1}\right)^{i j}\left(\mathbf{x}^{\mathbf{2}}\right)^{j i}+\delta\left(\mu^{2}\right)^{i j k}\left(\mathbf{x}^{\mathbf{3}}\right)^{k j i}-\left(\mu^{2}\right)^{i j k} \delta x^{k}\left(\mathbf{x}^{\mathbf{2}}\right)^{j i}+r^{i} \delta x^{i},
$$

and thanks to the fact that $\delta\left(\mu^{1}\right)^{i j}=\left(\mu^{2}\right)^{i j k} \delta x^{k}+\rho^{i j}$, we obtain:

$$
\delta\left[\mathcal{J}\left(r^{i} d x^{i}\right)\right]=\rho^{i j}\left(\mathbf{x}^{2}\right)^{j i}+\delta\left(\mu^{2}\right)^{i j k}\left(\mathbf{x}^{\mathbf{3}}\right)^{k j i}+r^{i} \delta x^{i} .
$$

Assuming now that $\rho^{i j}\left(\mathbf{x}^{\mathbf{2}}\right)^{j i}, \delta\left(\mu^{2}\right)^{i j k}\left(\mathbf{x}^{\mathbf{3}}\right)^{k j i}, r^{i} \delta x^{i} \in \mathcal{C}_{3}^{\nu}$ with $\nu>1$, then $\rho^{i j}\left(\mathbf{x}^{2}\right)^{j i}+$ $\delta\left(\mu^{2}\right)^{i j k}\left(\mathbf{x}^{\mathbf{3}}\right)^{k j i}+r^{i} \delta x^{i}$ becomes an element of $\operatorname{Dom}(\Lambda)$. Thus, applying $\Lambda$ to both sides of (24) and inserting the result into (21) we get the expression (16) of Proposition 3.2. This justifies the fact that (16) is a natural expression for $\mathcal{J}\left(m^{i} d x^{i}\right)$.

As in [15], the previous proposition has a straightforward multidimensional extension, which we state in the following corollary:

Corollary 3.3. Let $x$ be a process satisfying Hypothesis 3.1 and let $m \in \mathcal{Q}_{\kappa, b, c}\left(\mathbb{R}^{l, d}\right)$ with decomposition $m_{0}=b \in \mathbb{R}^{l, d}$ and

$$
\left(\delta m^{i j}\right)_{s t}=\left(\mu_{s}^{1}\right)^{i j k}\left(\delta x^{k}\right)_{s t}+\left(\mu_{s}^{2}\right)^{i j k_{1} k_{2}}\left(\mathbf{x}_{s t}^{2}\right)^{k_{2} k_{1}}+r_{s t}^{i j} ; \quad \delta\left(\mu_{s}^{1}\right)^{i j k_{1}}=\left(\mu_{s}^{2}\right)^{i j k_{1} k_{2}} \delta x^{k_{2}}+\rho^{i j k_{1}},
$$

where $\left(\mu^{1}\right)^{i j k_{1}} \in \mathcal{C}_{1}^{\kappa}(\mathbb{R}),\left(\mu^{2}\right)^{i j k_{1} k_{2}} \in \mathcal{C}_{1}^{\kappa}(\mathbb{R}), \rho^{i j k_{1}} \in \mathcal{C}_{1}^{2 \kappa}(\mathbb{R})$ and $r^{i j} \in \mathcal{C}_{2}^{3 \kappa}(\mathbb{R})$, for $i=$ $1, \ldots, l$ and $j, k_{1}, k_{2}=1, \ldots, d$. Define $z$ by $z_{0}=a \in \mathbb{R}^{l}$ and

$$
\begin{aligned}
\delta z^{i} & =\mathcal{J}\left(m^{i j} d x^{j}\right) \equiv m^{i j} \delta x^{j}+\left(\mu^{1}\right)^{i j k}\left(\mathbf{x}^{\mathbf{2}}\right)^{k j} \\
& +\left(\mu^{2}\right)^{i j k_{1} k_{2}}\left(\mathbf{x}^{\mathbf{3}}\right)^{k_{2} k_{1} j}+\Lambda\left(r^{i j} \delta x^{j}+\rho^{i j k}\left(\mathbf{x}^{\mathbf{2}}\right)^{k j}+\delta\left(\mu^{2}\right)^{i j k_{1} k_{2}}\left(\mathbf{x}^{\mathbf{3}}\right)^{k_{2} k_{1} j}\right) .
\end{aligned}
$$

Then the conclusions of Proposition 3.9 still hold in this context. 
We also observe that our extended pathwise integral has a nice continuity property with respect to the driving path $x$, whose proof is also skipped here for sake of conciseness (see also [9, Proposition 4], and [15, Proposition 3.12]).

Proposition 3.4. Let $x$ be a function satisfying Hypotheses 3.1 and 3.9. Suppose that there exists a sequence $\left\{x^{n} ; n \geq 1\right\}$ of piecewise $C^{1}$-functions from $[0, T]$ to $\mathbb{R}^{d}$ such that

$$
\lim _{n \rightarrow \infty} \mathcal{N}\left[x^{n}-x ; \mathcal{C}_{1}^{\gamma}\left(\mathbb{R}^{d}\right)\right]=0, \quad \lim _{n \rightarrow \infty} \mathcal{N}\left[\mathbf{x}^{2, n}-\mathbf{x}^{2} ; \mathcal{C}_{2}^{2 \gamma}\left(\mathbb{R}^{d, d}\right)\right]=0
$$

and $\lim _{n \rightarrow \infty} \mathcal{N}\left[\mathbf{x}^{\mathbf{3}, n}-\mathbf{x}^{\mathbf{3}} ; \mathcal{C}_{2}^{3 \gamma}\left(\mathbb{R}^{d, d, d}\right)\right]=0$. For $n \geq 1$, define $z^{n} \in \mathcal{C}_{1}^{\kappa}\left(\mathbb{R}^{l}\right)$ in the following way: set $z_{0}^{n}=b \in \mathbb{R}^{l}$ and assume that $\delta z^{n}$ can be decomposed into:

$$
\delta\left(z^{n}\right)^{i}=\left(\zeta^{1, n}\right)^{i j} \delta x^{j}+\left(\zeta^{2, n}\right)^{i j k}\left(\mathbf{x}^{2}\right)^{k j}+\left(r^{n}\right)^{i}, \quad \delta\left(\zeta^{1, n}\right)^{i j}=\left(\zeta^{2, n}\right)^{i j k} \delta x^{k}+\left(\rho^{n}\right)^{i j},
$$

for $1 \leq i \leq l$ and $1 \leq j, k \leq d$, where $\zeta^{1, n} \in \mathcal{C}_{1}^{\kappa}\left(\mathbb{R}^{l, d}\right)$ satisfies $\zeta_{0}^{1, n}=c \in \mathbb{R}^{l, d}$, and $\zeta^{2, n} \in \mathcal{C}_{1}^{\kappa}\left(\overline{\mathbb{R}^{l}, d, d}\right), \rho^{n} \in \mathcal{C}_{2}^{2 \kappa}\left(\mathbb{R}^{l, d}\right)$ and $r^{n} \in \mathcal{C}_{2}^{3 \kappa}\left(\mathbb{R}^{l}\right)$. Let also $z$ be a weakly controlled process with decomposition (10), such that $z_{0}=b, \zeta_{0}^{1}=c$, and suppose that

$$
\begin{aligned}
\lim _{n \rightarrow \infty}\{ & \mathcal{N}\left[z^{n}-z ; \mathcal{C}_{1}^{\kappa}\left(\mathbb{R}^{l}\right)\right]+\mathcal{N}\left[\zeta^{1, n}-\zeta^{1} ; \mathcal{C}_{1}^{\infty}\left(\mathbb{R}^{l, d}\right)\right]+\mathcal{N}\left[\zeta^{1, n}-\zeta^{1} ; \mathcal{C}_{1}^{\kappa}\left(\mathbb{R}^{l, d}\right)\right] \\
& +\mathcal{N}\left[\zeta^{2, n}-\zeta^{2} ; \mathcal{C}_{1}^{\infty}\left(\mathbb{R}^{l, d, d}\right)\right]+\mathcal{N}\left[\zeta^{2, n}-\zeta^{2} ; \mathcal{C}_{1}^{\kappa}\left(\mathbb{R}^{l, d, d}\right)\right] \\
& \left.+\mathcal{N}\left[\rho^{n}-\rho ; \mathcal{C}_{2}^{2 \kappa}\left(\mathbb{R}^{l, d}\right)\right]+\mathcal{N}\left[r^{n}-r ; \mathcal{C}_{2}^{3 \kappa}\left(\mathbb{R}^{l}\right)\right]\right\}=0
\end{aligned}
$$

Finally, let $\varphi: \mathbb{R}^{l} \rightarrow \mathbb{R}^{l^{\prime}, d}$ be a $C_{b}^{4}$-function. Then

$$
\lim _{n \rightarrow \infty} \mathcal{N}\left[\mathcal{J}\left(\varphi\left(z^{n}\right) d x^{n}\right)-\mathcal{J}(\varphi(z) d x) ; \mathcal{C}_{2}^{\kappa}\left(\mathbb{R}^{l^{\prime}}\right)\right]=0 .
$$

3.4. Rough diffusions equations. In this section, we shall apply the previous considerations to study differential equations driven by a rough signal, and recall that we first wish to solve simple equations of the form

$$
d y_{t}=\sigma\left(y_{t}\right) d x_{t}, \quad y_{0}=a,
$$

where $t \in[0, T], y$ is a $\mathbb{R}^{l}$-valued continuous process, $\sigma: \mathbb{R}^{l} \rightarrow \mathbb{R}^{l, d}$ is a smooth enough function, $x$ is a $\mathbb{R}^{d}$-valued path and $a \in \mathbb{R}^{l}$ is a fixed initial condition.

In our algebraic setting, we rephrase equation (27) as follows: we shall say that $y$ is a solution to (27), if $y_{0}=a, y \in \mathcal{Q}_{\kappa, a, \sigma(a)}\left(\mathbb{R}^{l}\right)$ and for any $0 \leq s \leq t \leq T$ we have

$$
(\delta y)_{s t}=\mathcal{J}_{s t}(\sigma(y) d x)
$$

where the integral $\mathcal{J}(\sigma(y) d x)$ has to be understood in the sense of Corollary 3.3.

With these notations in mind, our existence and uniqueness result is the following:

Theorem 3.5. Let $x$ be a process satisfying Hypotheses 3.1 and 3.9, and $\sigma: \mathbb{R}^{l} \rightarrow \mathbb{R}^{l, d}$ be a $C_{b}^{4}$-function. Then

(i) Equation (28) admits a unique solution y in $\mathcal{Q}_{\kappa, a, \sigma(a)}\left(\mathbb{R}^{l}\right)$ for any $\kappa<\gamma$ such that $3 \kappa+\gamma>1$.

(ii) The mapping $\left(a, x, \mathbf{x}^{\mathbf{2}}, \mathbf{x}^{\mathbf{3}}\right) \mapsto y$ is continuous from

$$
\mathbb{R}^{l} \times \mathcal{C}_{1}^{\gamma}\left(\mathbb{R}^{d}\right) \times \mathcal{C}_{2}^{2 \gamma}\left(\mathbb{R}^{d, d}\right) \times \mathcal{C}_{2}^{3 \gamma}\left(\mathbb{R}^{d, d, d}\right) \text { to } \mathcal{C}_{1}^{\kappa}\left(\mathbb{R}^{l}\right),
$$

in the following sense: let $z$ be the unique solution of (28) in $\mathcal{Q}_{\kappa, a, \sigma(a)}\left(\mathbb{R}^{l}\right)$ and $\tilde{z}$ the unique solution of (28) in $\mathcal{Q}_{\kappa, \tilde{a}, \sigma(\tilde{a})}\left(\mathbb{R}^{l}\right)$, based on $x, \tilde{x}$, respectively. Then, there 
exists a positive constant $\hat{c}_{\sigma, x, \tilde{x}}$ depending only on $\sigma, x, \tilde{x}$ such that

$$
\begin{aligned}
\mathcal{N}\left[z-\tilde{z} ; \mathcal{C}_{1}^{\kappa}\left(\mathbb{R}^{l}\right)\right] \leq \hat{c}_{x, \tilde{x}}\{|a-\tilde{a}|+ & \mathcal{N}\left[x-\tilde{x} ; \mathcal{C}_{1}^{\gamma}\left(\mathbb{R}^{l}\right)\right] \\
& \left.+\mathcal{N}\left[\mathbf{x}^{2}-\tilde{\mathbf{x}}^{2} ; \mathcal{C}_{2}^{2 \gamma}\left(\mathbb{R}^{d, d}\right)\right]+\mathcal{N}\left[\mathbf{x}^{3}-\tilde{\mathbf{x}}^{3} ; \mathcal{C}_{2}^{3 \gamma}\left(\mathbb{R}^{d, d, d}\right)\right]\right\} .
\end{aligned}
$$

Proof. As in [9, 11], we first identify the solution on a small interval $[0, \tau]$ as the fixed point of the map $\Gamma: \mathcal{Q}_{\kappa, a, \sigma(a)}\left(\mathbb{R}^{l}\right) \rightarrow \mathcal{Q}_{\kappa, a, \sigma(a)}\left(\mathbb{R}^{l}\right)$ defined by $\Gamma(z)=\hat{z}$ with $\hat{z}_{0}=a$ and $\delta \hat{z}=\mathcal{J}(\sigma(z) d x)$. The first step in this direction is to show that the ball

$$
B_{M}=\left\{z ; z_{0}=a, \mathcal{N}\left[z ; \mathcal{Q}_{\kappa, a, \sigma(a)}\left([0, \tau] ; \mathbb{R}^{l}\right)\right] \leq M\right\}
$$

is invariant under $\Gamma$ if $\tau$ is small enough and $M$ is large enough. However, due to Corollary 3.3 and Proposition 3.1, invoking the fact that $\sigma$ is bounded together with its derivaties and assuming $\tau \leq 1$, we obtain

$$
\begin{aligned}
\mathcal{N}\left[\Gamma(z) ; \mathcal{Q}_{\kappa, a, \sigma(a)}\left(\mathbb{R}^{l}\right)\right] & \leq c_{x}\left\{1+|\sigma(a)|_{\mathbb{R}^{l, d}}+\tau^{\gamma-\kappa}\left(|\sigma(a)|_{\mathbb{R}^{l, d}}+\mathcal{N}\left[\sigma(z) ; \mathcal{Q}_{\kappa, \hat{a}, \hat{b}}\left(\mathbb{R}^{l, d}\right)\right]\right)\right\} \\
& \leq c_{x, \sigma}\left\{1+\tau^{\gamma-\kappa} \mathcal{N}\left[\sigma(z) ; \mathcal{Q}_{\kappa, \hat{a}, \hat{b}}\left(\mathbb{R}^{l, d}\right)\right]\right\} \\
& \leq c_{x, \sigma}\left\{1+\tau^{\gamma-\kappa}\left(1+\mathcal{N}^{3}\left[z ; \mathcal{Q}_{\kappa, a, \sigma(a)}\left(\mathbb{R}^{l}\right)\right]\right)\right\} \\
& \leq \tilde{c}_{x, \sigma}\left\{1+\tau^{\gamma-\kappa} \mathcal{N}^{3}\left[z ; \mathcal{Q}_{\kappa, a, \sigma(a)}\left(\mathbb{R}^{l}\right)\right]\right\}
\end{aligned}
$$

where $\hat{a}=\sigma(a)$ and $\hat{b}=\partial_{i} \sigma(a) \sigma^{i \cdot}(a)$. Taking $M>\tilde{c}_{x, \sigma}$ and $\tau \leq \tau_{0}=\left(\frac{1}{M^{2} \tilde{c}_{x, \sigma}}-\frac{1}{M^{3}}\right)^{\frac{1}{\gamma-\kappa}} \wedge 1$, we obtain that $\tilde{c}_{x, \sigma}\left(1+\tau^{\gamma-\kappa} M^{3}\right) \leq M$. Therefore, the ball $B_{M}$ defined at (29) is left invariant by $\Gamma$.

It is now a matter of standard considerations to settle a fixed point argument for $\Gamma$ on $[0, \tau]$, and also to patch solutions on any interval of the form $[k \tau,(k+1) \tau]$ for $k \geq 1$. The details of this procedure are left to the reader.

\section{The Delay EQuation CASE}

This section is devoted to show how to change the diffusion setting in order to cover the case of delayed systems, having in mind to solve an equation of the form:

$$
\left\{\begin{array}{l}
d y_{t}=\sigma\left(y_{t}, y_{t-r_{1}}, \ldots, y_{t-r_{q}}\right) d x_{t} \quad t \in[0, T] \\
y_{t}=\xi_{t}, \quad t \in\left[-r_{q}, 0\right]
\end{array}\right.
$$

where $x$ is $\mathbb{R}^{d}$-valued $\gamma$-Hölder continuous function with $\gamma>1 / 4$, the function $\sigma$ is smooth enough, $\xi$ is a $\mathbb{R}^{n}$-valued $3 \gamma$-Hölder continuous function, and $0<r_{1}<\ldots<r_{q}<\infty$. Notice that for notational convenience, we set $r_{0}=0$ and we shall also use the notation

$$
\mathfrak{s}(y)_{t}=\left(y_{t-r_{1}}, \ldots, y_{t-r_{q}}\right), \quad t \in[0, T],
$$

which means that equation (31) can be written as:

$$
\left\{\begin{array}{l}
d y_{t}=\sigma\left(y_{t-r_{0}}, \mathfrak{s}(y)_{t}\right) d x_{t} \quad t \in[0, T] \\
y_{t}=\xi_{t}, \quad t \in\left[-r_{q}, 0\right]
\end{array}\right.
$$

As in [16], the main ingredient in order to go from the diffusion to the delayed case will be the introduction of a new class of processes, namely the class of delayed controlled paths, which captures the structure of our equation. We shall thus first define this new class of paths, and see how to integrate them with respect to the driving process $x$. 
4.1. Delayed controlled paths. As in the diffusion case, our analysis will rely on some a priori increments based on our driving noise $x$. More specifically, we set $\delta(x(v))_{s t} \triangleq$ $(\delta x)_{s-v, t-v}$ for $s, t, v \in[0, T]$, and we assume the following:

Hypothesis 4.1. The path $x$ is a $\mathbb{R}^{d}$-valued $\gamma$-Hölder continuous function with $\gamma>$ $1 / 4$, and admits two doubly delayed Lévy areas and two doubly delayed volume elements. Namely, for $v, v^{\prime} \in\left\{r_{q}, \ldots, r_{0}\right\}$, we assume that there exist four paths

$$
\mathbf{x}^{2}\left(v^{\prime}, v\right), \mathbf{x}^{2}\left(v^{\prime}-v, v\right) \in \mathcal{C}_{2}^{2 \gamma}\left([0, T] ; \mathbb{R}^{d, d}\right), \quad \mathbf{x}^{\mathbf{3}}\left(v^{\prime}, v\right), \mathbf{x}^{\mathbf{3}}\left(v^{\prime}-v, v\right) \in \mathcal{C}_{2}^{3 \gamma}\left([0, T] ; \mathbb{R}^{d, d, d}\right),
$$

satisfying the relations $\delta \mathbf{x}^{\mathbf{2}}\left(v^{\prime \prime}, v\right)=\delta\left(x\left(v+v^{\prime \prime}\right)\right) \otimes \delta(x(v))$ and

$$
\delta \mathbf{x}^{\mathbf{3}}\left(v^{\prime \prime}, v\right)=\mathbf{x}^{2}\left(v^{\prime \prime}, v\right) \otimes \delta x+\delta\left(x\left(v+v^{\prime \prime}\right)\right) \otimes \mathbf{x}^{\mathbf{2}}\left(v, r_{0}\right),
$$

which can also be written as:

$$
\begin{aligned}
\left(\delta\left(\mathbf{x}^{2}\left(v^{\prime \prime}, v\right)\right)^{i j}\right)_{s u t} & =\left(\delta x^{i}\right)_{s-v-v^{\prime \prime}, u-v-v^{\prime \prime}}\left(\delta x^{j}\right)_{u-v, t-v} \\
\left(\delta\left(\mathbf{x}^{\mathbf{3}}\left(v^{\prime \prime}, v\right)\right)^{i j k}\right)_{s u t} & =\left(\mathbf{x}_{s u}^{2}\left(v^{\prime \prime}, v\right)\right)^{i j}\left(\delta x^{k}\right)_{u t}+\left(\delta x^{i}\right)_{s-v-v^{\prime \prime}, u-v-v^{\prime \prime}}\left(\mathbf{x}_{u t}^{2}\left(v, r_{0}\right)\right)^{j k},
\end{aligned}
$$

for $v^{\prime \prime}=v^{\prime}$ or $v^{\prime}-v$, for any $s, u, t \in[0, T]$, and any $i, j, k \in\{1, \ldots, d\}$. The following notational simplification will also be used in the sequel: we may set $\mathbf{x}^{2}\left(v^{\prime \prime}\right):=\mathbf{x}^{\mathbf{2}}\left(v^{\prime \prime}, v\right)$ whenever $v=r_{0}$.

Remark 4.1. This hypothesis takes a more complex form than in [16], where the case $\gamma>1 / 3$ was treated. However, in case of a regular process $x$, it should be noticed that the increments $\mathbf{x}^{\mathbf{2}}\left(v_{1}, v_{2}\right)$ and $\mathbf{x}^{\mathbf{3}}\left(v_{1}, v_{2}\right)$ can be defined as:

$$
\mathbf{x}_{s t}^{2}\left(v_{1}, v_{2}\right)=\int_{s-v_{2}}^{t-v_{2}}\left(\delta x\left(v_{1}\right)\right)_{s-v_{2}, w} \otimes d x_{w}, \quad \text { and } \quad \mathbf{x}_{s t}^{3}\left(v_{1}, v_{2}\right)=\int_{s}^{t} \mathbf{x}_{s w}^{2}\left(v_{1}, v_{2}\right) \otimes d x_{w},
$$

which means that $\mathbf{x}^{\mathbf{2}}$ (resp. $\mathbf{x}^{\mathbf{3}}$ ) takes the usual form of a double (resp. triple) iterated integral.

As in Hypothesis 3.2, one should also express the fact that products of increments can be expressed in terms of iterated integrals. The following hypothesis is then easily shown to be a natural extension of what can be obtained in case of a smooth function $x$ :

Hypothesis 4.2. For $v, v^{\prime} \in\left\{r_{q}, \ldots, r_{0}\right\}$, let $\mathbf{x}^{2}\left(v^{\prime}, v\right)$ and $\mathbf{x}^{2}\left(v^{\prime}-v, v\right)$ be the area processes defined at Hypothesis 4.1 . Then we suppose that for all $0 \leq s<t \leq T$, we have $\mathbf{x}_{s t}^{2}\left(v^{\prime}, v\right)=\mathbf{x}_{s-v, t-v}^{2}\left(v^{\prime}, r_{0}\right)$ and

$$
[\delta x(v)]_{s t} \otimes\left[\delta x\left(v^{\prime}\right)\right]_{s t}=\mathbf{x}_{s t}^{2}\left(v-v^{\prime}, v^{\prime}\right)+\left(\mathbf{x}_{s t}^{2}\left(v^{\prime}-v, v\right)\right)^{*}
$$

With these hypotheses in hand, the delay equation will be solved in the space of delayed controlled processes, which can be defined as follows:

Definition 4.1. Let $-\infty<a<b \leq T$, a given initial datum $\alpha \in \mathbb{R}^{n}$ and $z \in \mathcal{C}_{1}^{\kappa}\left([a, b] ; \mathbb{R}^{n}\right)$ with $\kappa \leq \gamma$ and $3 \kappa+\gamma>1$. We say that $z$ is a delayed controlled path based on $x$ if $z_{a}=\alpha$, and if $\delta z \in \mathcal{C}_{2}^{\kappa}\left([a, b] ; \mathbb{R}^{n}\right)$ can be decomposed into

$$
\delta z^{i}=\left(\zeta^{1}\right)^{i j} \delta x^{j}+\left(\zeta^{\left(2, i^{\prime}\right)}\right)^{i j k}\left(\mathbf{x}^{2}\left(r_{i^{\prime}}\right)\right)^{k j}+\mathcal{R}^{i},
$$

for all $1 \leq i \leq n$, and where the index $i^{\prime}$ is summed over the set $0 \leq i^{\prime} \leq q$. Just as in Definition 3.1, the process $\zeta^{1}$ above has to admit the further decomposition: $\zeta_{a}^{1}=\beta \in \mathbb{R}^{n, d}$, where $\beta$ has to be interpreted as another initial datum, and for $1 \leq j, k \leq d$

$$
\delta\left(\zeta^{1}\right)^{i j}=\left(\zeta^{\left(2, i^{\prime}\right)}\right)^{i j k} \delta\left(x\left(r_{i^{\prime}}\right)\right)^{k}+\rho^{i j} .
$$


The regularity of the processes introduced above has to be the following: $\zeta^{1}$ is an element of $\mathcal{C}_{1}^{\kappa}\left([a, b] ; \mathbb{R}^{n, d}\right), \zeta^{\left(2, i^{\prime}\right)} \in \mathcal{C}_{1}^{\kappa}\left([a, b] ; \mathbb{R}^{n, d, d}\right)$, and the remainders $\mathcal{R}, \rho$ must satisfy $\mathcal{R} \in$ $\mathcal{C}_{2}^{3 \kappa}\left([a, b] ; \mathbb{R}^{n}\right)$ and $\rho \in \mathcal{C}_{2}^{2 \kappa}\left([a, b] ; \mathbb{R}^{n, d}\right)$.

The space of delayed controlled paths on $[a, b]$ will be denoted by $\mathcal{D}_{\kappa, \alpha, \beta}\left([a, b] ; \mathbb{R}^{n}\right)$, and a path $z \in \mathcal{D}_{\kappa, \alpha, \beta}\left([a, b] ; \mathbb{R}^{n}\right)$ should be considered in fact as a $(q+3)$-tuple $\left(z, \zeta^{1}, \zeta^{(2,0)}, \ldots\right.$, $\left.\zeta^{(2, q)}\right)$. The natural semi-norm on $\mathcal{D}_{\kappa, \alpha, \beta}\left([a, b] ; \mathbb{R}^{n}\right)$ is then given by

$$
\begin{aligned}
& \mathcal{N}\left[z ; \mathcal{D}_{\kappa, \alpha, \beta}\left([a, b] ; \mathbb{R}^{n}\right)\right] \\
& =\mathcal{N}\left[z ; \mathcal{C}_{1}^{\kappa}\left([a, b] ; \mathbb{R}^{n}\right)\right]+\mathcal{N}\left[\zeta^{1} ; \mathcal{C}_{1}^{\infty}\left([a, b] ; \mathbb{R}^{n, d}\right)\right]+\mathcal{N}\left[\zeta^{1} ; \mathcal{C}_{1}^{\kappa}\left([a, b] ; \mathbb{R}^{n, d}\right)\right] \\
& +\sum_{i^{\prime}=0}^{q} \mathcal{N}\left[\zeta^{\left(2, i^{\prime}\right)} ; \mathcal{C}_{1}^{\infty}\left([a, b] ; \mathbb{R}^{n, d, d}\right)\right]+\sum_{i^{\prime}=0}^{q} \mathcal{N}\left[\zeta^{\left(2, i^{\prime}\right)} ; \mathcal{C}_{1}^{\kappa}\left([a, b] ; \mathbb{R}^{n, d, d}\right)\right] \\
& +\mathcal{N}\left[\rho ; \mathcal{C}_{2}^{2 \kappa}\left([a, b] ; \mathbb{R}^{n, d}\right)\right]+\mathcal{N}\left[\mathcal{R} ; \mathcal{C}_{2}^{3 \kappa}\left([a, b] ; \mathbb{R}^{n}\right)\right]
\end{aligned}
$$

where we recall that the notations $\mathcal{N}\left[g ; \mathcal{C}_{1}^{\kappa}([a, b] ; V)\right]$ and $\mathcal{N}\left[g ; \mathcal{C}_{1}^{\infty}([a, b] ; V)\right]$ have been introduced at Section 2.2.

Unfortunately, the structure above is not sufficient in order to solve the fractional delay equation for $\gamma \leq 1 / 3$, and an additional notion of doubly delayed controlled processes has to be introduced.

Definition 4.2. Let $-\infty<a<b \leq T$, a given initial datum $\hat{\alpha} \in \mathbb{R}^{n}$ and $z \in \mathcal{C}_{1}^{\kappa}\left([a, b] ; \mathbb{R}^{n}\right)$ with $\kappa \leq \gamma$ and $3 \kappa+\gamma>1$. We say that $z$ is a doubly delayed controlled path based on $x$, if $z_{a}=\hat{\alpha}$ and if $\delta z \in \mathcal{C}_{2}^{\kappa}\left([a, b] ; \mathbb{R}^{n}\right)$ can be decomposed into

$$
\begin{aligned}
\delta z^{i}=\left(\zeta^{\left(1, i^{\prime \prime}\right)}\right)^{i j} \delta\left(x\left(r_{i^{\prime \prime}}\right)\right)^{j}+\left(\zeta^{\left(2, i^{\prime}, j^{\prime}\right)}\right)^{i j k}\left(\mathbf{x}^{2}\left(r_{j^{\prime}}, r_{i^{\prime}}\right)\right)^{k j} \\
\\
+\left(\zeta^{\left(3, i^{\prime \prime}, j^{\prime \prime}\right)}\right)^{i j k}\left(\mathbf{x}^{2}\left(r_{j^{\prime \prime}}-r_{i^{\prime \prime}}, r_{i^{\prime \prime}}\right)\right)^{k j}+\mathcal{R}^{i}
\end{aligned}
$$

for all $1 \leq i \leq n, 1 \leq j, k \leq d$, and where the indices $i^{\prime}, j^{\prime}$ and $i^{\prime \prime}, j^{\prime \prime}$ are summed over the set $\{1, \ldots, q\}$ and $\{0,1, \ldots, q\}$, respectively. As in Definition $\mathbf{4 . 1}$, the processes $\zeta^{\left(1, i^{\prime \prime}\right)}$ above have to admit the further decomposition: $\zeta_{a}^{\left(1, i^{\prime \prime}\right)}=\hat{\beta}^{\left(i^{\prime \prime}\right)}$, where $\hat{\beta}^{\left(i^{\prime \prime}\right)} \in \mathbb{R}^{n, d}$ has to be interpreted as another initial datum, and

$$
\begin{aligned}
& \delta\left(\zeta^{(1,0)}\right)^{i j}=\left(\zeta^{\left(3,0, j^{\prime \prime}\right)}\right)^{i j k} \delta\left(x\left(r_{j^{\prime \prime}}\right)\right)^{k}+\left(\rho^{(0)}\right)^{i j}, \\
& \delta\left(\zeta^{\left(1, i^{\prime}\right)}\right)^{i j}=\left(\zeta^{\left(2, i^{\prime}, j^{\prime}\right)}\right)^{i j k} \delta\left(x\left(r_{i^{\prime}}+r_{j^{\prime}}\right)\right)^{k}+\left(\zeta^{\left(3, i^{\prime}, j^{\prime \prime}\right)}\right)^{i j k} \delta\left(x\left(r_{j^{\prime \prime}}\right)\right)^{k}+\left(\rho^{\left(i^{\prime}\right)}\right)^{i j} .
\end{aligned}
$$

The regularity we ask for the processes introduced above is the following: $\zeta^{\left(1, i^{\prime \prime}\right)}$ is an element of $\mathcal{C}_{1}^{\kappa}\left([a, b] ; \mathbb{R}^{n, d}\right)$, we have $\zeta^{\left(2, i^{\prime}, j^{\prime}\right)}, \zeta^{\left(3, i^{\prime \prime}, j^{\prime \prime}\right)} \in \mathcal{C}_{1}^{\kappa}\left([a, b] ; \mathbb{R}^{n, d, d}\right)$, and the remainders $\mathcal{R}, \rho^{\left(i^{\prime \prime}\right)}$ satisfy $\mathcal{R} \in \mathcal{C}_{2}^{3 \kappa}\left([a, b] ; \mathbb{R}^{n}\right)$ and $\rho^{\left(i^{\prime \prime}\right)} \in \mathcal{C}_{2}^{2 \kappa}\left([a, b] ; \mathbb{R}^{n, d}\right)$.

The space of doubly delayed controlled paths on the interval $[a, b]$ will be denoted by $\widehat{\mathcal{D}}_{\kappa, \hat{\alpha}, \hat{\beta}}\left([a, b] ; \mathbb{R}^{n}\right)$, and a path $z \in \widehat{\mathcal{D}}_{\kappa, \hat{\alpha}, \hat{\beta}}\left([a, b] ; \mathbb{R}^{n}\right)$ should be considered in fact as a $\left(2 q^{2}+\right.$ $3 q+3)$-tuple $\left(z,\left\{\zeta^{\left(1, i^{\prime \prime}\right)}\right\},\left\{\zeta^{\left(2, i^{\prime}, j^{\prime}\right)}\right\},\left\{\zeta^{\left(3, i^{\prime \prime}, j^{\prime \prime}\right)}\right\}\right)$. The natural semi-norm on $\widehat{\mathcal{D}}_{\kappa, \hat{\alpha}, \hat{\beta}}\left([a, b] ; \mathbb{R}^{n}\right)$ 
is given by

$$
\begin{aligned}
& \mathcal{N}\left[z ; \widehat{\mathcal{D}}_{\kappa, \hat{\alpha}, \hat{\beta}}\left([a, b] ; \mathbb{R}^{n}\right)\right] \\
& =\mathcal{N}\left[z ; \mathcal{C}_{1}^{\kappa}\left([a, b] ; \mathbb{R}^{n}\right)\right]+\sum_{i^{\prime}=0}^{q} \mathcal{N}\left[\zeta^{\left(1, i^{\prime}\right)} ; \mathcal{C}_{1}^{\infty}\left([a, b] ; \mathbb{R}^{n, d}\right)\right]+\sum_{i^{\prime}=0}^{q} \mathcal{N}\left[\zeta^{\left(1, i^{\prime}\right)} ; \mathcal{C}_{1}^{\kappa}\left([a, b] ; \mathbb{R}^{n, d}\right)\right] \\
& +\sum_{i^{\prime}, j^{\prime}=1}^{q} \mathcal{N}\left[\zeta^{\left(2, i^{\prime}, j^{\prime}\right)} ; \mathcal{C}_{1}^{\infty}\left([a, b] ; \mathbb{R}^{n, d, d}\right)\right]+\sum_{i^{\prime}, j^{\prime}=1}^{q} \mathcal{N}\left[\zeta^{\left(2, i^{\prime}, j^{\prime}\right)} ; \mathcal{C}_{1}^{\kappa}\left([a, b] ; \mathbb{R}^{n, d, d}\right)\right] \\
& +\sum_{i^{\prime}, j^{\prime}=0}^{q} \mathcal{N}\left[\zeta^{\left(3, i^{\prime}, j^{\prime}\right)} ; \mathcal{C}_{1}^{\infty}\left([a, b] ; \mathbb{R}^{n, d, d}\right)\right]+\sum_{i^{\prime}, j^{\prime}=0}^{q} \mathcal{N}\left[\zeta^{\left(3, i^{\prime}, j^{\prime}\right)} ; \mathcal{C}_{1}^{\kappa}\left([a, b] ; \mathbb{R}^{n, d, d}\right)\right] \\
& +\mathcal{N}\left[\rho ; \mathcal{C}_{2}^{2 \kappa}\left([a, b] ; \mathbb{R}^{n, d}\right)\right]+\mathcal{N}\left[\mathcal{R} ; \mathcal{C}_{2}^{3 \kappa}\left([a, b] ; \mathbb{R}^{n}\right)\right]
\end{aligned}
$$

As in Section B, we shall now see how delayed controlled paths behave under composition with a smooth map, and also how to integrate them with respect to the driving process $x$.

4.2. Composition of delayed controlled processes. The composition of a delayed process with a smooth map like the function $\sigma$ appearing in equation (31) gives raise to a doubly delayed controlled process. This fact is detailed in the following proposition, for which we recall a notation: for a function $\varphi:\left(\mathbb{R}^{n}\right)^{q+1} \rightarrow \mathbb{R}$, we denote by $\partial_{i}^{j} \varphi\left(w_{0}, w_{1}, \ldots, w_{q}\right)$ the derivative of $\varphi$ with respect to the $i^{\text {th }}$ component of $w_{j}$, for $i \leq n$ and $j=0, \ldots, q$.

Proposition 4.1. Assume Hypothesis 4.9 holds true. Consider $0 \leq a<b \leq T$, let $\alpha, \tilde{\alpha}$ and $\beta, \tilde{\beta}$ be two initial conditions, respectively in $\mathbb{R}^{n}$ and $\mathbb{R}^{n, d}$, and let also $\varphi$ be a function in $C_{b}^{3}\left(\mathbb{R}^{n, q+1} ; \mathbb{R}\right)$. Define a map $T_{\varphi}$ on $\mathcal{D}_{\kappa, \alpha, \beta}\left([a, b] ; \mathbb{R}^{n}\right) \times \mathcal{D}_{\kappa, \tilde{\alpha}, \tilde{\beta}}\left(\left[a-r_{q}, b-r_{1}\right] ; \mathbb{R}^{n}\right)$ by $T_{\varphi}(z, \tilde{z})=\hat{z}$, with $\hat{z}_{t}=\varphi\left(z_{t}, \mathfrak{s}(\tilde{z})_{t}\right)$, for all $t \in[a, b]$. Then, setting

$$
\hat{\alpha}=\varphi\left(\alpha, \mathfrak{s}(\tilde{z})_{a}\right), \quad \hat{\beta}^{(0)}=\partial_{i}^{0} \varphi\left(\alpha, \mathfrak{s}(\tilde{z})_{a}\right) \beta^{i}, \quad \hat{\beta}^{\left(i^{\prime}\right)}=\partial_{i}^{i^{\prime}} \varphi\left(\alpha, \mathfrak{s}(\tilde{z})_{a}\right)\left(\tilde{\zeta}_{a-r_{i^{\prime}}}^{1}\right)^{i},
$$

for all $1 \leq i^{\prime} \leq q$, we have that $T_{\varphi}(z, \tilde{z}) \in \widehat{\mathcal{D}}_{\kappa, \hat{\alpha}, \hat{\beta}}([a, b] ; \mathbb{R})$. Moreover, the following cubic bound holds true:

$$
\begin{aligned}
& \mathcal{N}\left[\hat{z} ; \widehat{\mathcal{D}}_{\kappa, \hat{\alpha}, \hat{\beta}}([a, b] ; \mathbb{R})\right] \\
& \leq c_{\varphi, x, T}\left(1+\mathcal{N}^{3}\left[z ; \mathcal{D}_{\kappa, \alpha, \beta}\left([a, b] ; \mathbb{R}^{n}\right)\right]+\mathcal{N}^{3}\left[\tilde{z} ; \mathcal{D}_{\kappa, \tilde{\alpha}, \tilde{\beta}}\left(\left[a-r_{q}, b-r_{1}\right] ; \mathbb{R}^{n}\right)\right]\right)
\end{aligned}
$$

Proof. As in Proposition 3.1, the proof of this result is based on some cumbersome Taylor expansions which won't be detailed here. Let us just mention briefly the decomposition we obtain for $\hat{z}$ : observe that $\delta \hat{z}$ can be decomposed into

$$
\begin{aligned}
(\delta \hat{z})_{s t} & =\left(\hat{\zeta}_{s}^{\left(1, i^{\prime}\right)}\right)^{j}\left(\delta x^{j}\right)_{s-r_{i^{\prime}}, t-r_{i^{\prime}}}+\left(\hat{\zeta}_{s}^{\left(2, i^{\prime}, j^{\prime}\right)}\right)^{j k}\left(\mathbf{x}_{s t}^{2}\left(r_{j^{\prime}}, r_{i^{\prime}}\right)\right)^{k j} \mathbb{1}_{\left\{i^{\prime}, j^{\prime} \neq 0\right\}} \\
& +\left(\hat{\zeta}_{s}^{\left(3, i^{\prime}, j^{\prime}\right)}\right)^{j k}\left(\mathbf{x}_{s t}^{2}\left(r_{j^{\prime}}-r_{i^{\prime}}, r_{i^{\prime}}\right)\right)^{k j}+\hat{\mathcal{R}}_{s t},
\end{aligned}
$$

where we recall that Einstein's convention on repeated indices is used, and where $j, k$ are summed over the set $\{1, \ldots, d\}$, and $i^{\prime}, j^{\prime}$ over the set $\{0,1, \ldots, q\}$. Furthermore, the expression for the coefficients $\hat{\zeta}$ is given by:

$$
\begin{aligned}
\left(\hat{\zeta}_{s}^{\left(1, i^{\prime}\right)}\right)^{j} & =\left[\partial_{i}^{i^{\prime}} \varphi(z, \mathfrak{s}(\tilde{z})) \cdot\left(\tilde{\zeta}^{1}\left(r_{i^{\prime}}\right)\right)^{i j}\right]_{s}, \\
\left(\hat{\zeta}_{s}^{\left(2, i^{\prime}, j^{\prime}\right)}\right)^{j k} & =\left[\partial_{i}^{i^{\prime}} \varphi(z, \mathfrak{s}(\tilde{z})) \cdot\left(\tilde{\zeta}^{\left(2, j^{\prime}\right)}\left(r_{i^{\prime}}\right)\right)^{i j k}\right]_{s},
\end{aligned}
$$


and

$$
\begin{aligned}
\left(\hat{\zeta}_{s}^{\left(3, i^{\prime}, j^{\prime}\right)}\right)^{j k}= & {\left[\partial_{i_{1} i_{2}}^{i^{\prime} j^{\prime}} \varphi(z, \mathfrak{s}(\tilde{z})) \cdot\left(\tilde{\zeta}^{1}\left(r_{i^{\prime}}\right)\right)^{i_{1} j} \cdot\left(\tilde{\zeta}^{1}\left(r_{j^{\prime}}\right)\right)^{i_{2} k}\right]_{s} } \\
& +\left[\partial_{i}^{i^{\prime}} \varphi(z, \mathfrak{s}(\tilde{z}))\left(\tilde{\zeta}^{(2,0)}\left(r_{i^{\prime}}\right)\right)^{i j k}\right]_{s} \mathbb{1}_{\left\{i^{\prime}=j^{\prime}\right\}}+\left[\partial_{i}^{0} \varphi(z, \mathfrak{s}(\tilde{z}))\left(\zeta^{\left(2, j^{\prime}\right)}\right)^{i j k}\right]_{s} \mathbb{1}_{\left\{i^{\prime}=0, j^{\prime} \neq 0\right\}},
\end{aligned}
$$

with the convention that $\tilde{\zeta}^{1}\left(r_{0}\right) \triangleq \zeta^{1}$ and $\tilde{\zeta}^{\left(2, j^{\prime}\right)}\left(r_{0}\right) \triangleq \zeta^{\left(2, j^{\prime}\right)}$, respectively.

As far as the decomposition of $\left(\hat{\zeta}^{\left(1, i^{\prime}\right)}\right)^{j}$ is concerned, we obtain, for $1 \leq j \leq d$ :

$$
\begin{aligned}
\left(\delta\left(\hat{\zeta}^{\left(1, i^{\prime}\right)}\right)^{j}\right)_{s t}=\mathbb{1}_{\left\{i^{\prime}, j^{\prime} \neq 0\right\}}\left(\hat{\zeta}_{s}^{\left(2, i^{\prime}, j^{\prime}\right)}\right)^{j k}\left(\delta x^{k}\right)_{s-r_{i^{\prime}}-r_{j^{\prime}}, t-r_{i^{\prime}}-r_{j^{\prime}}} & \\
& +\left(\hat{\zeta}_{s}^{\left(3, i^{\prime}, j^{\prime}\right)}\right)^{j k}\left(\delta x^{k}\right)_{s-r_{j^{\prime}}, t-r_{j^{\prime}}}+\left(\hat{\rho}_{s t}^{\left(i^{\prime}\right)}\right)^{j}
\end{aligned}
$$

For sake of conciseness, we don't include the (long) expressions we have obtained for the remainders $\hat{\mathcal{R}}_{s t},\left(\hat{\rho}_{s t}^{\left(i^{\prime}\right)}\right)^{j}$ here. They are also obtained via a Taylor type expansion, and the relation $\mathbf{x}_{s t}^{\mathbf{2}}\left(v^{\prime}, v\right)=\mathbf{x}_{s-v, t-v}^{2}\left(v^{\prime}, r_{0}\right)$ (imposed by Hypothesis 4.2$)$ turns out to be useful at some points of the computations.

It should also be mentioned that, for a fixed $\tilde{z}$, the map $T_{\varphi}(\cdot, \tilde{z}): \mathcal{D}_{\kappa, \alpha, \beta}\left([a, b] ; \mathbb{R}^{n}\right) \rightarrow$ $\widehat{\mathcal{D}}_{\kappa, \hat{\alpha}, \hat{\beta}}([a, b] ; \mathbb{R})$ is locally Lipschitz continuous:

Proposition 4.2. Let the notation of Proposition 4.1 prevail, and suppose that $\varphi$ is a function in $C_{b}^{4}\left(\mathbb{R}^{n, q+1} ; \mathbb{R}\right)$. Let $0 \leq a<b \leq T$, let $z^{(1)}, z^{(2)} \in \mathcal{D}_{\kappa, \alpha, \beta}\left([a, b] ; \mathbb{R}^{n}\right)$ and let $\tilde{z} \in \mathcal{D}_{\kappa, \tilde{\alpha}, \tilde{\beta}}\left(\left[a-r_{k}, b-r_{1}\right] ; \mathbb{R}^{n}\right)$. Then,

$$
\begin{aligned}
& \mathcal{N}\left[T_{\varphi}\left(z^{(1)}, \tilde{z}\right)-T_{\varphi}\left(z^{(2)}, \tilde{z}\right) ; \widehat{\mathcal{D}}_{\kappa, 0,0}([a, b] ; \mathbb{R})\right] \\
& \leq c_{x, \varphi, T}\left(1+C\left(z^{(1)}, z^{(2)}, \tilde{z}\right)\right)^{3} \mathcal{N}\left[z^{(1)}-z^{(2)} ; \mathcal{D}_{\kappa, 0,0}\left([a, b] ; \mathbb{R}^{n}\right)\right]
\end{aligned}
$$

where

$$
\begin{aligned}
C\left(z^{(1)}, z^{(2)}, \tilde{z}\right)= & \mathcal{N}\left[\tilde{z} ; \mathcal{D}_{\kappa, \tilde{\alpha}, \tilde{\beta}}\left(\left[a-r_{k}, b-r_{1}\right] ; \mathbb{R}^{n}\right)\right] \\
& +\mathcal{N}\left[z^{(1)} ; \mathcal{D}_{\kappa, \alpha, \beta}\left([a, b] ; \mathbb{R}^{n}\right)\right]+\mathcal{N}\left[z^{(2)} ; \mathcal{D}_{\kappa, \alpha, \beta}\left([a, b] ; \mathbb{R}^{n}\right)\right]
\end{aligned}
$$

and the constant $c_{x, \varphi, T}$ depends only on $x, \varphi$ and $T$.

4.3. Integration of delayed controlled paths. As we have seen in the previous section, the composition with a smooth enough function $\varphi$ transforms a delayed controlled path into a doubly delayed controlled path. We shall see now that the integration with respect to $x$ is acting in the other direction:

Proposition 4.3. For a given $\gamma>1 / 4$ and $\kappa \leq \gamma$, let $x$ be a path satisfying Hypothesis 4.1. Moreover, let $m \in \widehat{\mathcal{D}}_{\kappa, \hat{\alpha}, \hat{\beta}}\left([a, b] ; \mathbb{R}^{1, d}\right)$ such that the increments of $m$ are given by (35). Define $z$ by $z_{a}=\alpha \in \mathbb{R}$ and

$$
\begin{aligned}
(\delta z)_{s t}= & m_{s}^{i}\left(\delta x^{i}\right)_{s t}+\left(\zeta_{s}^{\left(1, i^{\prime \prime}\right)}\right)^{i j}\left(\mathbf{x}_{s t}^{2}\left(r_{i^{\prime \prime}}\right)\right)^{j i} \\
& +\left(\zeta_{s}^{\left(2, i^{\prime}, j^{\prime}\right)}\right)^{i j k}\left(\mathbf{x}_{s t}^{\mathbf{3}}\left(r_{j^{\prime}}, r_{i^{\prime}}\right)\right)^{k j i}+\left(\zeta_{s}^{\left(3, i^{\prime \prime}, j^{\prime \prime}\right)}\right)^{i j k}\left(\mathbf{x}_{s t}^{\mathbf{3}}\left(r_{j^{\prime \prime}}-r_{i^{\prime \prime}}, r_{i^{\prime \prime}}\right)\right)^{k j i}+\Lambda_{s t}(U),
\end{aligned}
$$

for $a \leq s<t \leq b, 1 \leq i^{\prime}, j^{\prime} \leq q, 0 \leq i^{\prime \prime}, j^{\prime \prime} \leq q$, and where $U$ is the increment defined by:

$$
\begin{aligned}
U=\mathcal{R}^{i} \delta x^{i}+\left(\rho^{\left(i^{\prime \prime}\right)}\right)^{i j}\left(\mathbf{x}^{2}\left(r_{i^{\prime \prime}}\right)\right)^{j i}+\delta\left(\zeta^{\left(2, i^{\prime}, j^{\prime}\right)}\right)^{i j k}\left(\mathbf{x}^{\mathbf{3}}\left(r_{j^{\prime}}, r_{i^{\prime}}\right)\right)^{k j i} & \\
& +\delta\left(\zeta^{\left(3, i^{\prime \prime}, j^{\prime \prime}\right)}\right)^{i j k}\left(\mathbf{x}^{\mathbf{3}}\left(r_{j^{\prime \prime}}-r_{i^{\prime \prime}}, r_{i^{\prime \prime}}\right)\right)^{k j i} .
\end{aligned}
$$

Finally, set

$$
\mathcal{J}\left(m^{i} d x^{i}\right)=\delta z
$$


Then,

(i) $\mathcal{J}\left(m^{i} d x^{i}\right)$ coincides with the usual Riemann integral, whenever $m$ and $x$ are smooth functions.

(ii) The path $z$ is well-defined as an element of $\mathcal{D}_{\kappa, \alpha, \beta}([a, b] ; \mathbb{R})$, with an initial condition $z_{a}=\alpha, m_{a}=\beta=\hat{\alpha}$ and a decomposition of the form

$$
\begin{aligned}
(\delta z)_{s t} & =m^{i} \delta x^{i}+\left(\zeta_{s}^{\left(1, i^{\prime \prime}\right)}\right)^{i j}\left(\mathbf{x}_{s t}^{2}\left(r_{i^{\prime \prime}}\right)\right)^{j i}+\hat{\mathcal{R}}_{s t}, \\
\left(\delta m^{i}\right)_{s t} & =\left(\zeta_{s}^{\left(1, i^{\prime \prime}\right)}\right)^{i j}\left(\delta x^{j}\right)_{s-r_{i^{\prime \prime}}, t-r_{i^{\prime \prime}}}+\hat{\rho}_{s t}^{i},
\end{aligned}
$$

where the remainders $\hat{\mathcal{R}} \in \mathcal{C}_{2}^{3 \kappa}([a, b] ; \mathbb{R})$ and $\hat{\rho} \in \mathcal{C}_{2}^{2 \kappa}\left([a, b] ; \mathbb{R}^{1, d}\right)$ are given by

$$
\begin{aligned}
\hat{\mathcal{R}}_{s t} & =\left(\zeta_{s}^{\left(2, i^{\prime}, j^{\prime}\right)}\right)^{i j k}\left(\mathbf{x}_{s t}^{3}\left(r_{j^{\prime}}, r_{i^{\prime}}\right)\right)^{k j i}+\left(\zeta_{s}^{\left(3, i^{\prime \prime}, j^{\prime \prime}\right)}\right)^{i j k}\left(\mathbf{x}_{s t}^{\mathbf{3}}\left(r_{j^{\prime \prime}}-r_{i^{\prime \prime}}, r_{i^{\prime \prime}}\right)\right)^{k j i}+\Lambda_{s t}(U), \\
\hat{\rho}_{s t}^{i} & =\left(\zeta_{s}^{\left(2, i^{\prime}, j^{\prime}\right)}\right)^{i j k}\left(\mathbf{x}_{s t}^{2}\left(r_{j^{\prime}}, r_{i^{\prime}}\right)\right)^{k j}+\left(\zeta_{s}^{\left(3, i^{\prime \prime}, j^{\prime \prime}\right)}\right)^{i j k}\left(\mathbf{x}_{s t}^{2}\left(r_{j^{\prime \prime}}-r_{i^{\prime \prime}}, r_{i^{\prime \prime}}\right)\right)^{k j}+\mathcal{R}_{s t}^{i},
\end{aligned}
$$

respectively.

(iii) The semi-norm of $z$ in $\mathcal{D}_{\kappa, \alpha, \beta}([a, b] ; \mathbb{R})$ can be estimated as

$$
\begin{aligned}
& \mathcal{N}\left[z ; \mathcal{D}_{\kappa, \alpha, \beta}([a, b] ; \mathbb{R})\right] \\
& \leq c_{x, T, \kappa, \gamma}\left\{1+|\hat{\alpha}|_{\mathbb{R}^{1, d}}+(b-a)^{\gamma-\kappa}\left(|\hat{\alpha}|_{\mathbb{R}^{1, d}}+\mathcal{N}\left[m ; \widehat{\mathcal{D}}_{\kappa, \hat{\alpha}, \hat{\beta}}\left([a, b] ; \mathbb{R}^{1, d}\right)\right]\right)\right\} .
\end{aligned}
$$

Furthermore, the following bound also holds true:

$$
\|\delta z\|_{\kappa} \leq c_{x, T, \kappa, \gamma}(b-a)^{\gamma-\kappa}\left(|\hat{\alpha}|_{\mathbb{R}^{1, d}}+\mathcal{N}\left[m ; \widehat{\mathcal{D}}_{\kappa, \hat{\alpha}, \hat{\beta}}\left([a, b] ; \mathbb{R}^{1, d}\right)\right]\right) .
$$

(iv) The Riemann type sums associated to our generalized integral are of the following form:

$$
\begin{aligned}
\mathcal{J}_{s t}\left(m^{i} d x^{i}\right)=\lim _{\left|\Pi_{s t}\right| \rightarrow 0} \sum_{k^{\prime}=0}^{N}\left[m_{t_{k^{\prime}}}^{i}\left(\delta x^{i}\right)_{t_{k^{\prime}}, t_{k^{\prime}+1}}+\left(\zeta_{t_{k^{\prime}}}^{\left(1, i^{\prime \prime}\right)}\right)^{i j}\left(\mathbf{x}_{t_{k^{\prime}}, t_{k^{\prime}+1}}^{2}\left(r_{i^{\prime \prime}}\right)\right)^{j i}\right. \\
\left.\quad+\left(\zeta_{t_{k^{\prime}}}^{\left(2, i^{\prime}, j^{\prime}\right)}\right)^{i j k}\left(\mathbf{x}_{t_{k^{\prime}}, t_{k^{\prime}+1}}^{3}\left(r_{j^{\prime}}, r_{i^{\prime}}\right)\right)^{k j i}+\left(\zeta_{t_{k^{\prime}}}^{\left(3, i^{\prime \prime}, j^{\prime \prime}\right)}\right)^{i j k}\left(\mathbf{x}_{t_{k^{\prime}}, t_{k^{\prime}+1}}^{3}\left(r_{j^{\prime \prime}}-r_{i^{\prime \prime}}, r_{i^{\prime \prime}}\right)\right)^{k j i}\right],
\end{aligned}
$$

for any $a \leq s<t \leq b$, where the limit is taken over any partitions $\Pi_{s t}=\left\{t_{0}=s, \ldots, t_{n}=\right.$ $t\}$ of $[s, t]$, as the mesh of the partition goes to zero.

Proof. As in the proof of Proposition 3.2, we shall mainly focus on the first of these assertions, which justifies our definition of generalized integral. Let us thus suppose for the moment that $x$ is a smooth function and that $m \in \mathcal{C}_{1}^{\infty}\left(\mathbb{R}^{1, d}\right)$ admits the decomposition (35) with $\zeta^{\left(1, i^{\prime \prime}\right)} \in \mathcal{C}_{1}^{\infty}\left(\mathbb{R}^{d, d}\right), \zeta^{\left(2, i^{\prime}, j^{\prime}\right)}, \zeta^{\left(3, i^{\prime \prime}, j^{\prime \prime}\right)} \in \mathcal{C}_{1}^{\infty}\left(\mathbb{R}^{d, d, d}\right)$, for $1 \leq i^{\prime}, j^{\prime} \leq q$ and $0 \leq i^{\prime \prime}, j^{\prime \prime} \leq q$. We also assume that $\rho^{\left(i^{\prime \prime}\right)} \in \mathcal{C}_{2}^{\infty}\left(\mathbb{R}^{d, d}\right)$ and $\mathcal{R} \in \mathcal{C}_{2}^{\infty}\left(\mathbb{R}^{1, d}\right)$. Then $\mathcal{J}\left(m^{i} d x^{i}\right)$ is well-defined as a Riemann integral, and as for relation (21), one can write

$$
\mathcal{J}\left(m^{i} d x^{i}\right)=m^{i} \delta x^{i}+\mathcal{J}\left(\delta m^{i} d x^{i}\right) .
$$

Let us now plug the decomposition (35) into the expression (47) in order to obtain

$$
\begin{aligned}
\mathcal{J}\left(m^{i} d x^{i}\right)= & m^{i} \delta x^{i}+\left(\zeta^{\left(1, i^{\prime \prime}\right)}\right)^{i j}\left(\mathbf{x}^{\mathbf{2}}\left(r_{i^{\prime \prime}}\right)\right)^{j i}+\left(\zeta^{\left(2, i^{\prime}, j^{\prime}\right)}\right)^{i j k}\left(\mathbf{x}^{\mathbf{3}}\left(r_{j^{\prime}}, r_{i^{\prime}}\right)\right)^{k j i} \\
& +\left(\zeta^{\left(3, i^{\prime \prime}, j^{\prime \prime}\right)}\right)^{i j k}\left(\mathbf{x}^{\mathbf{3}}\left(r_{j^{\prime \prime}}-r_{i^{\prime \prime}}, r_{i^{\prime \prime}}\right)\right)^{k j i}+\mathcal{J}\left(\mathcal{R}^{i} d x^{i}\right),
\end{aligned}
$$

which can also be written as:

$$
\begin{aligned}
\mathcal{J}\left(\mathcal{R}^{i} d x^{i}\right)= & \mathcal{J}\left(m^{i} d x^{i}\right)-m^{i} \delta x^{i}-\left(\zeta^{\left(1, i^{\prime \prime}\right)}\right)^{i j}\left(\mathbf{x}^{2}\left(r_{i^{\prime \prime}}\right)\right)^{j i} \\
& -\left(\zeta^{\left(2, i^{\prime}, j^{\prime}\right)}\right)^{i j k}\left(\mathbf{x}^{\mathbf{3}}\left(r_{j^{\prime}}, r_{i^{\prime}}\right)\right)^{k j i}-\left(\zeta^{\left(3, i^{\prime \prime}, j^{\prime \prime}\right)}\right)^{i j k}\left(\mathbf{x}^{\mathbf{3}}\left(r_{j^{\prime \prime}}-r_{i^{\prime \prime}}, r_{i^{\prime \prime}}\right)\right)^{k j i}
\end{aligned}
$$


We now apply the operator $\delta$ to both sides of the previous equation in order to get a suitable expression for a generalization to the rough case:

$$
\begin{aligned}
\delta\left[\mathcal{J}\left(\mathcal{R}^{i} d x^{i}\right)\right]= & \delta m^{i} \delta x^{i}+\delta\left(\zeta^{\left(1, i^{\prime \prime}\right)}\right)^{i j}\left(\mathbf{x}^{2}\left(r_{i^{\prime \prime}}\right)\right)^{j i}-\left(\zeta^{\left(1, i^{\prime \prime}\right)}\right)^{i j}\left[\delta\left(x\left(r_{i^{\prime \prime}}\right)\right)^{j} \delta x^{i}\right] \\
& +\delta\left(\zeta^{\left(2, i^{\prime}, j^{\prime}\right)}\right)^{i j k}\left(\mathbf{x}^{\mathbf{3}}\left(r_{j^{\prime}}, r_{i^{\prime}}\right)\right)^{k j i}+\delta\left(\zeta^{\left(3, i^{\prime \prime}, j^{\prime \prime}\right)}\right)^{i j k}\left(\mathbf{x}^{\mathbf{3}}\left(r_{j^{\prime \prime}}-r_{i^{\prime \prime}}, r_{i^{\prime \prime}}\right)\right)^{k j i} \\
& -\left(\zeta^{\left(2, i^{\prime}, j^{\prime}\right)}\right)^{i j k}\left[\left(\mathbf{x}^{2}\left(r_{j^{\prime}}, r_{i^{\prime}}\right)\right)^{k j} \delta x^{i}+\delta\left(x\left(r_{i^{\prime}}+r_{j^{\prime}}\right)\right)^{k}\left(\mathbf{x}^{\mathbf{2}}\left(r_{i^{\prime}}\right)\right)^{j i}\right] \\
& -\left(\zeta^{\left(3, i^{\prime \prime}, j^{\prime \prime}\right)}\right)^{i j k}\left[\left(\mathbf{x}^{\mathbf{2}}\left(r_{j^{\prime \prime}}-r_{i^{\prime \prime}}, r_{i^{\prime \prime}}\right)\right)^{k j} \delta x^{i}+\delta\left(x\left(r_{j^{\prime \prime}}\right)\right)^{k}\left(\mathbf{x}^{2}\left(r_{i^{\prime \prime}}\right)\right)^{j i}\right] .
\end{aligned}
$$

Taking now into account the decomposition (35) for $m$ and (36) for $\zeta^{\left(1, i^{\prime \prime}\right)}$, we obtain:

$$
\begin{aligned}
\delta\left[\mathcal{J}\left(\mathcal{R}^{i} d x^{i}\right)\right]=\mathcal{R}^{i} \delta x^{i}+\left(\rho^{\left(i^{\prime \prime}\right)}\right)^{i j}\left(\mathbf{x}^{2}\left(r_{i^{\prime \prime}}\right)\right)^{j i} & +\delta\left(\zeta^{\left(2, i^{\prime}, j^{\prime}\right)}\right)^{i j k}\left(\mathbf{x}^{\mathbf{3}}\left(r_{j^{\prime}}, r_{i^{\prime}}\right)\right)^{k j i} \\
& +\delta\left(\zeta^{\left(3, i^{\prime \prime}, j^{\prime \prime}\right)}\right)^{i j k}\left(\mathbf{x}^{\mathbf{3}}\left(r_{j^{\prime \prime}}-r_{i^{\prime \prime}}, r_{i^{\prime \prime}}\right)\right)^{k j i}=U
\end{aligned}
$$

where $U$ is defined by equation (42). If we now only assume that $U$ is an element of $\mathcal{C}_{3}^{\mu}$ with $\mu>1$, one gets that $U \in \operatorname{Dom}(\Lambda)$, which yields the decomposition (43) for $\mathcal{J}\left(m^{i} d x^{i}\right)$. The remainder of the proof is then just made of tedious elementary estimations for the regularity of all the terms involved in the decomposition (43). Finally, observe that the Riemann sum limit is obtained by applying Corollary 2.3.

As in Corollary 3.3, the previous Proposition can be extended easily to the multidimensional case:

Corollary 4.4. Let $m \in \widehat{\mathcal{D}}_{\kappa, \hat{\alpha}, \hat{\beta}}\left([a, b] ; \mathbb{R}^{n, d}\right)$ be a doubly delayed controlled path, and define another path $z$ by $\delta z^{i}=\mathcal{J}\left(m^{i j} d x^{j}\right)$ for $i=1, \ldots, n$. Then $z$ is well-defined as an element of $\mathcal{D}_{\kappa, \alpha, \beta}\left([a, b] ; \mathbb{R}^{n}\right)$, and the following bound holds true:

$$
\begin{aligned}
& \mathcal{N}\left[z ; \mathcal{D}_{\kappa, \alpha, \beta}\left([a, b] ; \mathbb{R}^{n}\right)\right] \\
& \leq c_{x, T, \kappa, \gamma}\left\{1+|\hat{\alpha}|_{\mathbb{R}^{n, d}}+(b-a)^{\gamma-\kappa}\left(|\hat{\alpha}|_{\mathbb{R}^{n, d}}+\mathcal{N}\left[m ; \widehat{\mathcal{D}}_{\kappa, \hat{\alpha}, \hat{\beta}}\left([a, b] ; \mathbb{R}^{n, d}\right)\right]\right)\right\} .
\end{aligned}
$$

Another useful feature of our generalized integral is a continuity property with respect to the integrand $m$ in $\mathcal{J}(z d x)$, whose proof is omitted again for sake of conciseness:

Proposition 4.5. Let $m^{(1)}, m^{(2)} \in \widehat{\mathcal{D}}_{\kappa, \hat{\alpha}, \hat{\beta}}\left([a, b] ; \mathbb{R}^{n, d}\right)$ be two doubly delayed controlled paths, and define $z^{(1)}, z^{(2)} \in \mathcal{D}_{\kappa, \alpha, \beta}\left([a, b] ; \mathbb{R}^{n}\right)$ by $\delta z^{(l)}=\mathcal{J}\left(m^{(l)} d x\right)$, for $l=1,2$. Assume moreover that the paths $\zeta^{\left(1, i^{\prime \prime} ; 1\right)}$ and $\zeta^{\left(1, i^{\prime \prime} ; 2\right)}$ in the respective decompositions (35) of $z^{(1)}$ and $z^{(2)}$ satisfy $\zeta_{a}^{\left(1, i^{\prime \prime} ; 1\right)}=\zeta_{a}^{\left(1, i^{\prime \prime} ; 2\right)}$, and that $b-a<1$. Then we have:

$$
\begin{aligned}
\mathcal{N}\left[z^{(1)}-z^{(2)} ; \mathcal{D}_{\kappa, 0,0}([a, b]\right. & \left.\left.; \mathbb{R}^{n}\right)\right] \\
& \leq c_{x, T, \kappa, \gamma}(b-a)^{\kappa \wedge(\gamma-\kappa)} \mathcal{N}\left[m^{(1)}-m^{(2)} ; \widehat{\mathcal{D}}_{\kappa, 0,0}\left([a, b] ; \mathbb{R}^{n, d}\right)\right] .
\end{aligned}
$$

4.4. Rough delay differential equations. We shall now turn to the main goal of this section, namely the resolution of equation (31), which can be written now as:

$$
(\delta y)_{s t}=\mathcal{J}_{s t}(\sigma(y, \mathfrak{s}(y)) d x), \quad s, t \in[0, T],
$$

with initial condition $y_{t}=\xi_{t}$ for $t \in[-r, 0]$, where we recall the convention (32) for $\mathfrak{s}$, and where the integral $\mathcal{J}$ has to be interpreted according to Proposition 4.3 .

Before stating our main result in this direction, let us introduce a natural map, called $\Gamma$, associated to our delay equation. It is defined by $\Gamma(z, \tilde{z}):=\hat{z}$, where (recalling the 
notation $T_{\sigma}$ introduced at Proposition 4.1) $\delta \hat{z}$ is given as $\delta \hat{z}=\mathcal{J}\left(T_{\sigma}(z, \tilde{z}) d x\right)$, on the following spaces:

$$
\Gamma: \mathcal{D}_{\kappa, \alpha, \beta}\left([a, b] ; \mathbb{R}^{n}\right) \times \mathcal{D}_{\kappa, \tilde{\alpha}, \tilde{\beta}}\left(\left[a-r_{q}, b-r_{1}\right] ; \mathbb{R}^{n}\right) \rightarrow \mathcal{D}_{\kappa, \alpha, \beta}\left([a, b] ; \mathbb{R}^{n}\right)
$$

for $0 \leq a<b \leq T$. In the previous definition, $\alpha, \tilde{\alpha}, \beta$ and $\tilde{\beta}$ stand for some initial conditions, with the additional compatibility condition $\beta=\sigma\left(\alpha, \tilde{z}_{a-r_{1}}, \ldots, \tilde{z}_{a-r_{q-1}}, \tilde{z}_{a-r_{q}}\right)$, which shall be satisfied in our delay equation context. Notice also that, from now on, we shall use the convention that $z_{s}=\tilde{z}_{s}=\hat{z}_{s}=\xi_{s}$ for $s \in\left[-r_{q}, 0\right]$. Since we have assumed $\xi \in \mathcal{C}_{1}^{3 \gamma}$, this means in particular that, on $\left[-r_{q}, 0\right]$, the paths $z, \tilde{z}, \hat{z}$ are still controlled processes, whose degenerate decomposition is only given by a remainder term. This allows to complete easily the definition of $\Gamma$ on intervals of the form $[a, b]$ with $a<r_{q}$.

Remark 4.2. As in [16], we could have handled the case of a controlled initial condition $\xi$. We did not consider this possibility here for sake of conciseness.

Let us gather now some useful relations concerning the operator $\Gamma$ we have just defined: first of all, by putting together inequalities (51) and (37), one obtains

$$
\begin{aligned}
\mathcal{N}\left[\Gamma(z, \tilde{z}) ; \mathcal{D}_{\kappa, \alpha, \beta}\left([a, b] ; \mathbb{R}^{n}\right)\right] \leq c_{\sigma, x, T, \kappa, \gamma} & \left(1+\mathcal{N}^{3}\left[\tilde{z} ; \mathcal{D}_{\kappa, \tilde{\alpha}, \tilde{\beta}}\left(\left[a-r_{q}, b-r_{1}\right] ; \mathbb{R}^{n}\right)\right]\right) \\
& \times\left(1+(b-a)^{\gamma-\kappa} \mathcal{N}^{3}\left[z ; \mathcal{D}_{\kappa, \alpha, \beta}\left([a, b] ; \mathbb{R}^{n}\right)\right]\right),
\end{aligned}
$$

which means that the semi-norm of the mapping $\Gamma$ is cubically bounded in terms of the semi-norm of $z$ and $\tilde{z}$.

Furthermore, let $z^{(1)}, z^{(2)} \in \mathcal{D}_{\kappa, \alpha, \beta}\left([a, b] ; \mathbb{R}^{n}\right)$ and $\tilde{z} \in \mathcal{D}_{\kappa, \tilde{\alpha}, \tilde{\beta}}\left(\left[a-r_{q}, b-r_{1}\right] ; \mathbb{R}^{n}\right)$. Then, if $b-a<1$, applying successively the inequalities (52) and (40), we get that

$$
\begin{aligned}
& \mathcal{N}\left[\Gamma\left(z^{(1)}, \tilde{z}\right)-\Gamma\left(z^{(2)}, \tilde{z}\right) ; \mathcal{D}_{\kappa, 0,0}\left([a, b] ; \mathbb{R}^{n}\right)\right] \\
& \leq \tilde{c}_{x, \sigma, T, \kappa, \gamma}\left(1+C\left(z^{(1)}, z^{(2)}, \tilde{z}\right)\right)^{3}(b-a)^{\kappa \wedge(\gamma-\kappa)} \mathcal{N}\left[z^{(1)}-z^{(2)} ; \mathcal{D}_{\kappa, 0,0}\left([a, b] ; \mathbb{R}^{n}\right)\right],
\end{aligned}
$$

where $C\left(z^{(1)}, z^{(2)}, \tilde{z}\right)$ is defined at (41). Therefore, for fixed $\tilde{z}$ the mappings $\Gamma(\cdot, \tilde{z})$ are locally Lipschitz continuous with respect to the semi-norm $\mathcal{N}\left[\cdot ; \mathcal{D}_{\kappa, 0,0}\left([a, b] ; \mathbb{R}^{n}\right)\right]$.

With these preliminary results in hand, we can now prove our main theorem on delay equations:

Theorem 4.6. Let $x$ be a path satisfying Hypotheses 4.1 and 4.9 , let $\sigma: \mathbb{R}^{n, q+1} \rightarrow \mathbb{R}^{n, d}$ be a $C_{b}^{4}$-function and let $\xi \in \mathcal{C}_{1}^{3 \gamma}\left([-r, 0] ; \mathbb{R}^{n}\right)$. Then

(i) Equation (53) admits a unique solution $y$ in $\mathcal{D}_{\kappa, \xi_{0}, \sigma_{r}\left(\xi_{0}\right)}\left([0, T] ; \mathbb{R}^{n}\right)$, for any $\kappa<\gamma$ such that $3 \kappa+\gamma>1$ and any $T>0$, where $\sigma_{r}\left(\xi_{0}\right) \triangleq \sigma\left(\xi_{0}, \xi_{-r_{1}}, \ldots, \xi_{-r_{q}}\right)$.

(ii) The map

$$
\left(\xi, x,\left\{\mathbf{x}^{\mathbf{2}}\left(r_{i^{\prime \prime}}\right)\right\}_{0 \leq i^{\prime \prime} \leq q},\left\{\mathbf{x}^{\mathbf{3}}\left(r_{j^{\prime}}, r_{i^{\prime}}\right)\right\}_{1 \leq i^{\prime}, j^{\prime} \leq q},\left\{\mathbf{x}^{\mathbf{3}}\left(r_{j^{\prime \prime}}-r_{i^{\prime \prime}}, r_{i^{\prime \prime}}\right)\right\}_{0 \leq i^{\prime \prime}, j^{\prime \prime} \leq q}\right) \mapsto y
$$

is locally Lipschitz continuous from

$$
C_{1}^{3 \gamma}\left([-r, 0] ; \mathbb{R}^{n}\right) \times \mathcal{C}_{1}^{\gamma}\left([0, T] ; \mathbb{R}^{d}\right) \times\left(\mathcal{C}_{2}^{2 \gamma}\left([0, T] ; \mathbb{R}^{d, d}\right)\right)^{q+1} \times\left(\mathcal{C}_{2}^{3 \gamma}\left([0, T] ; \mathbb{R}^{d, d, d}\right)\right)^{2 q^{2}+2 q+1}
$$

to $\mathcal{C}_{1}^{\kappa}\left([0, T] ; \mathbb{R}^{n}\right.$, in the following sense: let $\tilde{x}$ be another driving rough path with corresponding delayed Lévy area and doubly delayed volume element $\tilde{\mathbf{x}}^{\mathbf{2}}(v), \tilde{\mathbf{x}}^{\mathbf{3}}\left(v^{\prime \prime}, v\right)$, respectively, 
where $v^{\prime \prime}=v^{\prime}$ or $v^{\prime}-v$, with $v, v^{\prime} \in\left\{r_{q}, \ldots, r_{0}\right\}$, and $\tilde{\xi}$ another initial condition. Then, for every $M>0$, there exists a constant $K_{M}>0$ such that the upper bound

$$
\begin{aligned}
& \mathcal{N}\left[y-\tilde{y} ; \mathcal{C}_{1}^{\kappa}\left([0, T] ; \mathbb{R}^{n}\right)\right] \leq K_{M}\left\{\mathcal{N}\left[\xi-\tilde{\xi} ; \mathcal{C}_{1}^{3 \gamma}\left([0, T] ; \mathbb{R}^{n}\right)\right]+\mathcal{N}\left[x-\tilde{x} ; \mathcal{C}_{1}^{\gamma}\left([0, T] ; \mathbb{R}^{n}\right)\right]\right. \\
& \quad+\sum_{i^{\prime \prime}=0}^{q} \mathcal{N}\left[\mathbf{x}^{\mathbf{2}}\left(r_{i^{\prime \prime}}\right)-\tilde{\mathbf{x}}^{2}\left(r_{i^{\prime \prime}}\right) ; \mathcal{C}_{2}^{2 \gamma}\left([0, T] ; \mathbb{R}^{d, d}\right)\right] \\
& \quad+\sum_{i^{\prime}, j^{\prime}=1}^{q} \mathcal{N}\left[\mathbf{x}^{3}\left(r_{j^{\prime}}, r_{i^{\prime}}\right)-\tilde{\mathbf{x}}^{\mathbf{3}}\left(r_{j^{\prime}}, r_{i^{\prime}}\right) ; \mathcal{C}_{2}^{3 \gamma}\left([0, T] ; \mathbb{R}^{d, d, d}\right)\right] \\
& \left.\quad+\sum_{i^{\prime \prime}, j^{\prime \prime}=0}^{q} \mathcal{N}\left[\mathbf{x}^{\mathbf{3}}\left(r_{j^{\prime \prime}}-r_{i^{\prime \prime}}, r_{i^{\prime \prime}}\right)-\tilde{\mathbf{x}}^{\mathbf{3}}\left(r_{j^{\prime \prime}}-r_{i^{\prime \prime}}, r_{i^{\prime \prime}}\right) ; \mathcal{C}_{2}^{3 \gamma}\left([0, T] ; \mathbb{R}^{d, d, d}\right)\right]\right\} .
\end{aligned}
$$

holds for all tuples

$$
\begin{aligned}
& \left(\xi, x,\left\{\mathbf{x}^{\mathbf{2}}\left(r_{i^{\prime \prime}}\right)\right\}_{0 \leq i^{\prime \prime} \leq q},\left\{\mathbf{x}^{\mathbf{3}}\left(r_{j^{\prime}}, r_{i^{\prime}}\right)\right\}_{1 \leq i^{\prime}, j^{\prime} \leq q},\left\{\mathbf{x}^{\mathbf{3}}\left(r_{j^{\prime \prime}}-r_{i^{\prime \prime}}, r_{i^{\prime \prime}}\right)\right\}_{0 \leq i^{\prime \prime}, j^{\prime \prime} \leq q}\right), \\
& \left(\tilde{\xi}, \tilde{x},\left\{\tilde{\mathbf{x}}^{\mathbf{2}}\left(r_{i^{\prime \prime}}\right)\right\}_{0 \leq i^{\prime \prime} \leq q},\left\{\tilde{\mathbf{x}}^{\mathbf{3}}\left(r_{j^{\prime}}, r_{i^{\prime}}\right)\right\}_{1 \leq i^{\prime}, j^{\prime} \leq q},\left\{\tilde{\mathbf{x}}^{\mathbf{3}}\left(r_{j^{\prime \prime}}-r_{i^{\prime \prime}}, r_{i^{\prime \prime}}\right)\right\}_{0 \leq i^{\prime \prime}, j^{\prime \prime} \leq q}\right),
\end{aligned}
$$

satisfying the boundedness condition

$$
\begin{aligned}
& \mathcal{N}\left[\xi ; \mathcal{C}_{1}^{3 \gamma}\left([0, T] ; \mathbb{R}^{n}\right)\right]+\mathcal{N}\left[\tilde{\xi} ; \mathcal{C}_{1}^{3 \gamma}\left([0, T] ; \mathbb{R}^{n}\right)\right]+\mathcal{N}\left[x ; \mathcal{C}_{1}^{\gamma}\left([0, T] ; \mathbb{R}^{n}\right)\right]+\mathcal{N}\left[\tilde{x} ; \mathcal{C}_{1}^{\gamma}\left([0, T] ; \mathbb{R}^{n}\right)\right] \\
& +\sum_{i^{\prime \prime}=0}^{q} \mathcal{N}\left[\mathbf{x}^{2}\left(r_{i^{\prime \prime}}\right) ; \mathcal{C}_{2}^{2 \gamma}\left([0, T] ; \mathbb{R}^{d, d}\right)\right]+\sum_{i^{\prime \prime}=0}^{q} \mathcal{N}\left[\tilde{\mathbf{x}}^{2}\left(r_{i^{\prime \prime}}\right) ; \mathcal{C}_{2}^{2 \gamma}\left([0, T] ; \mathbb{R}^{d, d}\right)\right] \\
& +\sum_{i^{\prime}, j^{\prime}=1}^{q} \mathcal{N}\left[\mathbf{x}^{\mathbf{3}}\left(r_{j^{\prime}}, r_{i^{\prime}}\right) ; \mathcal{C}_{2}^{3 \gamma}\left([0, T] ; \mathbb{R}^{d, d, d}\right)\right]+\sum_{i^{\prime}, j^{\prime}=1}^{q} \mathcal{N}\left[\tilde{\mathbf{x}}^{\mathbf{3}}\left(r_{j^{\prime}}, r_{i^{\prime}}\right) ; \mathcal{C}_{2}^{3 \gamma}\left([0, T] ; \mathbb{R}^{d, d, d}\right)\right] \\
& +\sum_{i^{\prime \prime}, j^{\prime \prime}=0}^{q} \mathcal{N}\left[\mathbf{x}^{\mathbf{3}}\left(r_{j^{\prime \prime}}-r_{i^{\prime \prime}}, r_{i^{\prime \prime}}\right) ; \mathcal{C}_{2}^{3 \gamma}\left([0, T] ; \mathbb{R}^{d, d, d}\right)\right] \\
& +\sum_{i^{\prime \prime}, j^{\prime \prime}=0}^{q} \mathcal{N}\left[\tilde{\mathbf{x}}^{3}\left(r_{j^{\prime \prime}}-r_{i^{\prime \prime}}, r_{i^{\prime \prime}}\right) ; \mathcal{C}_{2}^{3 \gamma}\left([0, T] ; \mathbb{R}^{d, d, d}\right)\right] \leq M
\end{aligned}
$$

Proof. With the previous algebraic structures and computations in hand, the proof of this theorem follows the lines of [16, Theorem 4.2]. We shall give some hints for the proof of the existence-uniqueness result, which is based on a fixed point argument for the map $\Gamma$ defined above, for sake of completeness.

Without loss of generality suppose that $T=N r_{1}$, where we recall that $r_{1}$ is the smallest delay in (53). We shall construct the solution of our delay equation by induction over the intervals $\left[0, r_{1}\right],\left[0,2 r_{1}\right], \ldots,\left[0, N r_{1}\right]$.

Let us first show that equation (53) has a solution on the interval $\left[0, r_{1}\right]$. To this purpose, define

$$
\tilde{\tau}_{1}=\left(\frac{1}{c_{1} M_{1}^{2}}-\frac{1}{M_{1}^{3}}\right)^{\frac{1}{\gamma-\kappa}} \wedge r_{1} \wedge 1
$$

where $M_{1}>c_{1}=c_{\kappa, \gamma, \sigma, T}\left(1+\mathcal{N}^{3}\left[\xi ; \mathcal{C}_{1}^{3 \gamma}\left(\left[-r_{q}, 0\right] ; \mathbb{R}^{n}\right)\right]\right)$. In addition, choose $\tau_{1} \in\left[0, \tilde{\tau}_{1}\right]$ and $N_{1} \in \mathbb{N}$ such that $N_{1} \tau_{1}=r_{1}$, and define

$$
I_{k^{\prime}, 1}=\left[\left(k^{\prime}-1\right) \tau_{1}, k^{\prime} \tau_{1}\right], \quad k^{\prime}=1, \ldots, N_{1} .
$$


Finally, consider the following map: let $\Gamma_{1,1}: \mathcal{D}_{\kappa, \xi_{0}, \sigma_{r}\left(\xi_{0}\right)}\left(I_{1,1} ; \mathbb{R}^{n}\right) \rightarrow \mathcal{D}_{\kappa, \xi_{0}, \sigma_{r}\left(\xi_{0}\right)}\left(I_{1,1} ; \mathbb{R}^{n}\right)$ be given by $\hat{z}=\Gamma_{1,1}(z)$, where

$$
\left(\delta \hat{z}^{i}\right)_{s t}=\mathcal{J}_{s t}\left(T_{\sigma}^{i j}(z, \xi) d x^{j}\right)
$$

for $0 \leq s<t \leq \tau_{1}$. Notice then that if $z^{(1,1)}$ is a fixed point of the map $\Gamma_{1,1}$, then $z^{(1,1)}$ solves equation (53) on the interval $I_{1,1}$. Therefore, we shall prove that such a fixed point exists.

First, owing to (54) we get the estimate

$$
\mathcal{N}\left[\Gamma_{1,1}(z) ; \mathcal{D}_{\kappa, \xi_{0}, \sigma_{r}\left(\xi_{0}\right)}\left(I_{1,1} ; \mathbb{R}^{n}\right)\right] \leq c_{1}\left(1+\tau_{1}^{\gamma-\kappa} \mathcal{N}^{3}\left[z ; \mathcal{D}_{\kappa, \xi_{0}, \sigma_{r}\left(\xi_{0}\right)}\left(I_{1,1} ; \mathbb{R}^{n}\right)\right]\right) .
$$

Therefore, thanks to our previous choice of $\tau_{1}$, we obtain that the ball

$$
B_{M_{1}}=\left\{z \in \mathcal{D}_{\kappa, \xi_{0}, \sigma_{r}\left(\xi_{0}\right)}\left(I_{1,1} ; \mathbb{R}^{n}\right) ; \mathcal{N}\left[z ; \mathcal{D}_{\kappa, \xi_{0}, \sigma_{r}\left(\xi_{0}\right)}\left(I_{1,1} ; \mathbb{R}^{n}\right)\right] \leq M_{1}\right\}
$$

is invariant under $\Gamma_{1,1}$. On the other hand, by changing $\tau_{1}$ to a smaller value (and then $N_{1}$ accordingly) if necessary, observe that $\Gamma_{1,1}$ also is a contraction on $B_{M_{1}}$, see (55). Thus, applying the Fixed Point Theorem, it is easily shown that there exists a unique solution $z^{(1,1)}$ to equation (53) on the interval $I_{1,1}$.

If $\tau_{1}=r_{1}$, we have thus obtained the existence and uniqueness of a solution in the interval $\left[0, r_{1}\right]$. Otherwise, define the map

$$
\Gamma_{2,1}: \mathcal{D}_{\kappa, z_{r_{1}}^{(1,1)}, \sigma_{r}\left(z_{r_{1}}^{(1,1)}\right)}\left(I_{2,1} ; \mathbb{R}^{n}\right) \longrightarrow \mathcal{D}_{\kappa, z_{r_{1}}^{(1,1)}, \sigma_{r}\left(z_{r_{1}}^{(1,1)}\right)}\left(I_{2,1} ; \mathbb{R}^{n}\right)
$$

given by $\hat{z}=\Gamma_{2,1}(z)$, where $\sigma_{r}\left(z_{r_{1}}^{(1,1)}\right) \triangleq \sigma\left(z_{r_{1}}^{(1,1)}, \xi_{0}, \ldots, \xi_{-r_{q-1}}\right)$ and

$$
\left(\delta \hat{z}^{i}\right)_{s t}=\mathcal{J}_{s t}\left(T_{\sigma}^{i j}(z, \xi) d x^{j}\right)
$$

for $\tau_{1} \leq s<t \leq 2 \tau_{1}$. Since $\tau_{1}<r_{1}$, the following upper bound still holds true:

$$
\mathcal{N}\left[\Gamma_{2,1}(z) ; \mathcal{D}_{\kappa, z_{r_{1}}^{(1,1)}, \sigma_{r}\left(z_{r_{1}}^{(1,1)}\right)}\left(I_{2,1} ; \mathbb{R}^{n}\right)\right] \leq c_{1}\left(1+\tau_{1}^{\gamma-\kappa} \mathcal{N}^{3}\left[z ; \mathcal{D}_{\kappa, z_{r_{1}}(1,1)}, \sigma_{r}\left(z_{r_{1}}^{(1,1)}\right)\left(I_{2,1} ; \mathbb{R}^{n}\right)\right]\right)
$$

and we obtain, resorting to the same fixed point argument as above, the existence of a unique solution $z^{(2,1)}$ to equation (53) on the interval $I_{2,1}$. Repeating this step as often as necessary, which is possible since the estimates on the norms of the mappings $\Gamma_{l, 1}$, $l=1, \ldots, N_{1}$ are of the same type as (54), that is, the constant $c_{1}$ in (58) does not change according to the iteration step, we obtain that $z=\sum_{l=1}^{N_{1}} z^{(l, 1)} \mathbb{1}_{l, 1}$ is the unique solution to the equation (53) on the interval $\left[0, r_{1}\right]$.

The patching of solutions defined on different intervals of the form $I_{l, k}$ is then a slight elaboration of the computations corresponding to [16, Theorem 4.2], and this step is left to the reader. The continuity of the Itô map is follows also the steps of [16], except for the huge number of terms we have to deal with in the current situation. We prefer to omit this step for sake of conciseness.

\section{Application to the fractional Brownian motion}

All the previous constructions rely on the specific assumptions that we have made on the process $x$. In this section, we prove how our results can be applied to the fractional Brownian motion. More specifically, we first recall some basic definitions about fBm, and then define the delayed Lévy area $\mathbf{B}^{2}$. We shall then turn to the definition of the volume $\mathbf{B}^{3}$, which is the main difficulty in order to go from the case $H>1 / 3$ treated in [16] to our rougher situation. 
5.1. Basic facts on fractional Brownian motion. Recall that a $d$-dimensional $\mathrm{fBm}$ with Hurst parameter $H \in(0,1)$ defined on the real line is a centered Gaussian process

$$
B=\left\{B_{t}=\left(B_{t}^{1}, \ldots, B_{t}^{d}\right) ; t \in \mathbb{R}\right\}
$$

where $B^{1}, \ldots, B^{d}$ are $d$ independent 1-dimensional $\mathrm{fBm}$, that is, each $B^{i}$ is a centered Gaussian process with continuous sample paths and covariance function

$$
R_{H}(t, s)=\mathbb{E}\left(B_{t}^{i} B_{s}^{i}\right)=\frac{1}{2}\left(|t|^{2 H}+|s|^{2 H}-|t-s|^{2 H}\right),
$$

for all $i \in\{1, \ldots, d\}$. In the sequel, all the random variables we deal with are defined on a complete probability space $(\Omega, \mathcal{F}, \mathbb{P})$, and we assume that $\mathcal{F}$ is generated by the random variables $\left(B_{t} ; t \in \mathbb{R}\right)$. The fBm verifies the following two important properties:

- Scaling property: for any $c>0, B^{(c)}=c^{H} B \cdot / c$ is a $\mathrm{fBm}$,

- Stationarity property: for any $h \in \mathbb{R}, B_{+h}-B_{h}$ is a $\mathrm{fBm}$.

Notice that we work with a fBm indexed by $\mathbb{R}$ for sake of simplicity as in [16], since this allows some more elegant calculations for the definitions of the double delayed Lévy area and volume, respectively. Furthermore, since the case $H>1 / 2$ or the Brownian case $H=1 / 2$ are less demanding than the rougher case, we shall mainly focus in this section on the range of parameter $H<1 / 2$.

5.1.1. Gaussian structure of B. Let us give a few facts about the Gaussian structure of fractional Brownian motion, following Chapter 5 of [17]. All the considerations in this direction will concern a 1-dimensional $\mathrm{fBm} B$, which will be enough for our applications.

Let $\mathcal{E}$ be the set of step-functions on $\mathbb{R}$ with values in $\mathbb{R}$. Consider the Hilbert space $\mathcal{H}$ defined as the closure of $\mathcal{E}$ with respect to the scalar product induced by

$$
\left\langle\mathbb{1}_{\left[t, t^{\prime}\right]}, \mathbb{1}_{\left[s, s^{\prime}\right]}\right\rangle_{\mathcal{H}}=R_{H}\left(t^{\prime}, s^{\prime}\right)-R_{H}\left(t^{\prime}, s\right)-R_{H}\left(t, s^{\prime}\right)+R_{H}(t, s),
$$

for any $-\infty<s<s^{\prime}<+\infty$ and $-\infty<t<t^{\prime}<+\infty$, and where $R_{H}(t, s)$ is given by (59). The mapping

$$
\mathbb{1}_{\left[t, t^{\prime}\right]} \mapsto B_{t^{\prime}}-B_{t}
$$

can be extended to an isometry between $\mathcal{H}$ and the Gaussian space $H_{1}(B)$ associated with $B$. We denote this isometry by $\varphi \mapsto B(\varphi)$.

The spaces $\mathcal{H}$ and $H_{1}(B)$ can be characterized more precisely in the following way: first, we notice that a 1 -dimensional $\mathrm{fBm}$ defined on the real line, with $H \neq 1 / 2$, has the following integral representation in terms of a Wiener process $W$ defined on $\mathbb{R}$ (See 21, Proposition 7.2.6] for details):

$$
B_{t}=\frac{1}{C_{1}(H)} \int_{\mathbb{R}}\left[(t-s)_{+}^{H-1 / 2}-(-s)_{+}^{H-1 / 2}\right] d W_{s}, \quad t \in \mathbb{R}
$$

where

$$
C_{1}(H)=\left(\int_{0}^{\infty}\left[(1+s)^{H-1 / 2}-s^{H-1 / 2}\right]^{2} d s+\frac{1}{2 H}\right)^{1 / 2},
$$

and where $a_{+}$stands for the positive part of a real number $a$, namely $a_{+}=\mathbb{1}_{\mathbb{R}_{+}}(a) a$. Using the representation (60), the authors in [18] define the following stochastic integral 
of a deterministic function with respect to a 1-dimensional $\mathrm{fBm} B$ :

$$
\int_{\mathbb{R}} f(u) d B_{u}=\frac{\Gamma(H+1 / 2)}{C_{1}(H)} \begin{cases}\int_{\mathbb{R}}\left(\mathcal{D}_{-}^{1 / 2-H} f\right)(u) d W_{u}, & H<1 / 2, \\ \int_{\mathbb{R}}\left(\mathcal{I}_{-}^{H-1 / 2} f\right)(u) d W_{u}, & H>1 / 2,\end{cases}
$$

provided that the stochastic integral with respect to the Wiener process $W$ makes sense, and where

$$
\begin{aligned}
& \left(\mathcal{D}_{-}^{\alpha} f\right)(u)=\frac{\alpha}{\Gamma(1-\alpha)} \int_{0}^{\infty} \frac{f(r)-f(u+r)}{r^{1+\alpha}} d r \\
& \left(\mathcal{I}_{-}^{\alpha} f\right)(u)=\frac{1}{\Gamma(\alpha)} \int_{u}^{\infty} \frac{f(r)}{(r-u)^{1-\alpha}} d r
\end{aligned}
$$

for $0<\alpha<1$. The expressions (62) and (63) are respectively called right-sided fractional derivative and right-sided fractional integral on the whole real line. We remark that, in general,

We also notice that

$$
\left(\mathcal{D}_{-}^{\alpha} f\right)(u) \equiv \lim _{\varepsilon \rightarrow 0} \frac{\alpha}{\Gamma(1-\alpha)} \int_{\varepsilon}^{\infty} \frac{f(r)-f(u+r)}{r^{1+\alpha}} d r .
$$

$$
\left(\mathcal{I}_{-}^{\alpha}\left(\left(\mathcal{D}_{-}^{\alpha} f\right)\right)(u)=\left(\mathcal{D}_{-}^{\alpha}\left(\mathcal{I}_{-}^{\alpha} f\right)\right)(u)=f(u) .\right.
$$

When $f$ is a function defined on an interval $[a, b]$ with $-\infty<a<b<\infty$, extend $f$ by setting $f^{\star}=f \mathbb{1}_{[a, b]}$. Define then

$$
\begin{aligned}
& \left(\mathcal{D}_{-}^{\alpha} f^{\star}\right)(u)=\left(\mathcal{D}_{-b}^{\alpha} f\right)(u)=\frac{f(u)}{\Gamma(1-\alpha)(b-u)^{\alpha}}+\frac{\alpha}{\Gamma(1-\alpha)} \int_{u}^{b} \frac{f(u)-f(r)}{(r-u)^{1+\alpha}} d r, \\
& \left(\mathcal{I}_{-}^{\alpha} f^{\star}\right)(u)=\left(\mathcal{I}_{-b}^{\alpha} f\right)(u)=\frac{1}{\Gamma(\alpha)} \int_{u}^{b} \frac{f(r)}{(r-u)^{1-\alpha}} d r,
\end{aligned}
$$

for $0<\alpha<1, a<u<b$. The expressions (65) and (66) are respectively called rightsided fractional derivative and right-sided fractional integral on the interval $[a, b]$. In this context, as in the case of the whole line (see [20] for details and also [22]), the following relation holds true:

$$
\left(\mathcal{D}_{-b}^{\alpha} f\right)(u) \equiv \frac{f(u)}{\Gamma(1-\alpha)(b-u)^{\alpha}}+\lim _{\varepsilon \rightarrow 0} \frac{\alpha}{\Gamma(1-\alpha)} \int_{u+\varepsilon}^{b} \frac{f(u)-f(r)}{(r-u)^{1+\alpha}} d r .
$$

With these notations in hand, it is proved in 18 that the operator

$$
(\mathcal{K} f)(u) \equiv \frac{\Gamma(H+1 / 2)}{C_{1}(H)} \begin{cases}\left(\mathcal{D}_{-}^{1 / 2-H} f\right)(u), & H<1 / 2, \\ \left(\mathcal{I}_{-}^{H-1 / 2} f\right)(u), & H>1 / 2\end{cases}
$$

is an isometry between $\mathcal{H}$ and a closed subspace of $L^{2}(\mathbb{R})$. In fact,

$$
\langle\phi, \psi\rangle_{\mathcal{H}}=\langle\mathcal{K} \phi, \mathcal{K} \psi\rangle_{L^{2}(\mathbb{R})}
$$

for all $\phi, \psi \in \mathcal{H}$. This also allows to write $B(\varphi)$ as $W(\mathcal{K} \varphi)$ for any $\varphi \in \mathcal{H}$, where $W(\mathcal{K} \varphi)$ has to be interpreted as a Wiener integral with respect to the Gaussian measure $W$. In particular, we have:

$$
\mathbb{E}\left[|B(\varphi)|^{2}\right]=\|\varphi\|_{\mathcal{H}}=\|\mathcal{K} \varphi\|_{L^{2}(\mathbb{R})}
$$


5.1.2. Malliavin calculus with respect to the $f B m B$. Let $\mathcal{S}$ be the set of smooth cylindrical random variables of the form

$$
F=f\left(B\left(\varphi_{1}\right), \ldots, B\left(\varphi_{k}\right)\right), \quad \varphi_{i} \in \mathcal{H}, \quad i \in\{1, \ldots, k\},
$$

where $f \in C^{\infty}\left(\mathbb{R}^{d, k}, \mathbb{R}\right)$ is bounded with bounded derivatives. The derivative operator $D$ of a smooth cylindrical random variable of the above form is defined as the $\mathcal{H}$-valued random variable

$$
D F=\sum_{i=1}^{k} \frac{\partial f}{\partial x_{i}}\left(B\left(\varphi_{1}\right), \ldots, B\left(\varphi_{k}\right)\right) \varphi_{i} .
$$

This operator is closable from $L^{p}(\Omega)$ into $L^{p}(\Omega ; \mathcal{H})$. As usual, $\mathbb{D}^{1,2}$ denotes the closure of the set of smooth random variables with respect to the norm

$$
\|F\|_{1,2}^{2}=\mathbb{E}|F|^{2}+\mathbb{E}\|D F\|_{\mathcal{H}}^{2} .
$$

In particular, considering a $d$-dimensional $\operatorname{fBm}\left(B^{1}, \ldots, B^{d}\right)$, if $D^{B^{i}} F$ denotes the Malliavin derivative of $F \in \mathbb{D}_{B^{i}}^{1,2}$ with respect to $B^{i}$, where $\mathbb{D}_{B^{i}}^{1,2}$ denotes the corresponding Sobolev space, we have $D^{B^{i}} B_{t}^{j}=\delta_{i, j} \mathbb{1}_{(-\infty, t]}$ for $i, j=1, \ldots, d$, where $\delta_{i, j}$ denotes the Kronecker symbol.

The divergence operator $I$ is the adjoint of the derivative operator. If a random variable $\phi \in L^{2}(\Omega ; \mathcal{H})$ belongs to $\operatorname{dom}(I)$, the domain of the divergence operator, then $I(\phi)$ is defined by the duality relationship

$$
\mathbb{E}(F I(\phi))=\mathbb{E}\langle D F, \phi\rangle_{\mathcal{H}},
$$

for every $F \in \mathbb{D}^{1,2}$. In additon, let us recall two useful properties verified by $D$ and $I$ :

- If $\phi \in \operatorname{dom}(I)$ and $F \in \mathbb{D}^{1,2}$ such that $F \phi \in L^{2}(\Omega ; \mathcal{H})$, then we have the following integration by parts formula:

$$
I(F \phi)=F I(\phi)-\langle D F, \phi\rangle_{\mathcal{H}}
$$

- If $\phi \in \mathbb{D}^{1,2}(\mathcal{H}), D_{r} \phi \in \operatorname{dom}(I)$ for all $r \in \mathbb{R}$ and $\left\{I\left(D_{r} \phi\right)\right\}_{r \in \mathbb{R}}$ is an element of $L^{2}(\Omega ; \mathcal{H})$, then

$$
D_{r} I(\phi)=\phi_{r}+I\left(D_{r} \phi\right)
$$

One can relate the Malliavin derivatives with respect to $B$ and $W$ through the operator $\mathcal{K}$ defined above. Indeed, relation (64) shows that $\mathcal{K}$ is invertible. This allows to state, as in the case of a 1-dimensional fBm $B$ in an interval (see for example [17, Section 5.2] and also [1]), the following relations for the Malliavin derivative and divergence operators with respect to the processes $B$ and $W$ :

(i) For any $F \in \mathbb{D}_{W}^{1,2}=\mathbb{D}^{1,2}$, we have:

$$
\mathcal{K} D F=D^{W} F,
$$

where $D^{W}$ denotes the derivative operator with respect to the process $\mathrm{W}$, and $\mathbb{D}_{W}^{1,2}$ the corresponding Sobolev space.

(ii) $\operatorname{Dom}(I)=\mathcal{K}^{-1}\left(\operatorname{Dom}\left(I^{W}\right)\right)$, and for any $\mathcal{H}$-valued random variable $u$ in $\operatorname{Dom}(I)$ we have $I(u)=I^{W}(\mathcal{K} u)$, where $I^{W}$ denotes the divergence operator with respect to the process $W$. 
In addition, we have $\mathbb{D}^{1,2}(\mathcal{H})=\left(\mathcal{K}^{-1}\right)\left(\mathbb{L}^{1,2}\right)$, where $\mathbb{L}^{1,2}=\mathbb{D}^{1,2}\left(L^{2}(\mathbb{R})\right)$, and this space is included in $\operatorname{dom}\left(I^{W}\right)$. Making use of the notations $I^{W}(\phi)=\int_{R} \phi_{u} d W_{u}$ for any $\phi \in$ $\operatorname{dom}\left(I^{W}\right)$, and $I(\phi)=\int_{R} \phi_{u} d B_{u}$ for any $\phi \in \operatorname{dom}(I)$, we can write:

$$
\int_{\mathbb{R}} \phi_{u} d B_{u}=\int_{\mathbb{R}}(\mathcal{K} \phi)(u) d W_{u}
$$

This kind of relation also holds when one considers functions defined on an interval. Indeed, for some fixed $-\infty<a<b<\infty$, and $H<1 / 2$, relation (65) yields

$$
\int_{a}^{b} \phi_{u} d B_{u}=\int_{\mathbb{R}} \phi_{u} \mathbb{1}_{[a, b]}(u) d B_{u}=\int_{\mathbb{R}}\left(\mathcal{K}\left[\phi \mathbb{1}_{[a, b]}\right]\right)(u) d W_{u}=\int_{\mathbb{R}}\left(\mathcal{K}^{[a, b]} \phi\right)(u) d W_{u},
$$

where the operator $\mathcal{K}^{[a, b]}$ is defined by:

$$
\left(\mathcal{K}^{[a, b]} f\right)(u) \equiv \frac{\Gamma(H+1 / 2)}{C_{1}(H)}\left(\mathcal{D}_{-b}^{1 / 2-H} f\right)(u), \quad \text { for } \quad a<u<b,
$$

with $C_{1}(H)$ defined by (61). In case of an interval $[a, b]$, it should also be mentioned that an important subspace of integrable processes is the following: let $\mathcal{E}^{[a, b]}$ be the set of step-functions on $[a, b]$ with values in $\mathbb{R}$. As in [17, Subsection 5.2.3], we consider on this space the semi-norm

$$
\|\varphi\|_{\mathcal{H}_{K}([a, b])}^{2}=\int_{a}^{b} \frac{\varphi_{u}^{2}}{(b-u)^{1-2 H}} d u+\int_{a}^{b}\left(\int_{u}^{b} \frac{\left|\varphi_{r}-\varphi_{u}\right|}{(r-u)^{3 / 2-H}} d r\right)^{2} d u .
$$

Let $\mathcal{H}_{K}([a, b])$ be the Hilbert space defined as the closure of $\mathcal{E}^{[a, b]}$ with respect to the previous semi-norm. Then the space $\mathcal{H}_{K}([a, b])$ is continuously included in $\mathcal{H}$, and if $\phi \in \mathbb{D}^{1,2}\left(\mathcal{H}_{K}([a, b])\right)$, then $\phi \in \operatorname{Dom}(I)$.

5.1.3. Generalized stochastic integrals. The stochastic integrals we shall use in order to define our doubly delayed Lévy area and volume are defined, in a natural way, by RussoVallois' symmetric approximations, that is, for a given process $\phi$ :

$$
\int_{a}^{b} \phi_{w} d^{\circ} B_{w}^{i}=L^{2}-\lim _{\varepsilon \rightarrow 0} \frac{1}{2 \varepsilon} \int_{a}^{b} \phi_{w}\left(B_{w+\varepsilon}^{i}-B_{w-\varepsilon}^{i}\right) d w
$$

provided the limit exists. It is well known that the Russo-Vallois symmetric integral coincides with Young's integral for $H>1 / 2$, and with the classical Stratonovich integral in the Brownian case $H=1 / 2$. Since these two cases are not very demanding from a technical point of view, we will focus our efforts on the case $1 / 4<H<1 / 2$. This being said, for $v_{1} \in[-r, r], v_{2} \in[0, r]$, such that $v_{1}+v_{2} \geq 0$, we will try to define the increments $\mathbf{B}^{2}$ and $\mathbf{B}^{\mathbf{3}}$ as

$$
\begin{gathered}
\mathbf{B}_{s t}^{2}\left(v_{1}, v_{2}\right)=\int_{s-v_{2}}^{t-v_{2}} d^{\circ} B_{u} \otimes \int_{s-v_{2}-v_{1}}^{u-v_{1}} d^{\circ} B_{\tau}, \text { i.e. }\left(\mathbf{B}_{s t}^{2}\left(v_{1}, v_{2}\right)\right)^{i j}=\int_{s-v_{2}}^{t-v_{2}} d^{\circ} B_{u}^{j} \int_{s-v_{2}-v_{1}}^{u-v_{1}} d^{\circ} B_{\tau}^{i} \\
\mathbf{B}_{s t}^{\mathbf{3}}\left(v_{1}, v_{2}\right)=\int_{s}^{t} d^{\circ} B_{w} \otimes \int_{s-v_{2}}^{w-v_{2}} d^{\circ} B_{u} \otimes \int_{s-v_{2}-v_{1}}^{u-v_{1}} d^{\circ} B_{\tau}, \\
\text { i.e. }\left(\mathbf{B}_{s t}^{3}\left(v_{1}, v_{2}\right)\right)^{i j k}=\int_{s}^{t} d^{\circ} B_{w}^{k} \int_{s-v_{2}}^{w-v_{2}} d^{\circ} B_{u}^{j} \int_{s-v_{2}-v_{1}}^{u-v_{1}} d^{\circ} B_{\tau}^{i}, \quad(71)
\end{gathered}
$$

for all $i, j, k \in\{1, \ldots, d\}, 0 \leq s<t \leq T<\infty$.

Interestingly enough, one can establish the existence of symmetric integrals thanks to some Malliavin calculus criterions: 
Proposition 5.1. Let $\phi$ be a stochastic process such that $\phi \mathbb{1}_{[a, b]} \in \mathbb{D}^{1,2}\left(\mathcal{H}_{K}([a, b])\right)$, for all $-\infty<a<b<\infty$. Suppose also that

$$
\operatorname{Tr}_{[a, b]} D \phi:=L^{2}-\lim _{\varepsilon \rightarrow 0} \frac{1}{2 \varepsilon} \int_{a}^{b}\left\langle D \phi_{u}, \mathbb{1}_{[u-\varepsilon, u+\varepsilon]}\right\rangle_{\mathcal{H}} d u
$$

is an almost surely finite random variable. Then $\int_{a}^{b} \phi_{u} d^{\circ} B_{u}^{i}$ exists, and verifies

$$
\int_{a}^{b} \phi_{u} d^{\circ} B_{u}^{i}=I\left(\phi \mathbb{1}_{[a, b]}\right)+\operatorname{Tr}_{[a, b]} D \phi .
$$

Furthermore, the following algebraic relation is trivially satisfied for this kind of integrals:

Lemma 5.2. Let $\alpha=\left\{\alpha_{w}, w \in[a, b]\right\}$ be a stochastic process such that its symmetric Russo-Vallois integral with respect to a 1-dimensional fractional Brownian motion $B$ exists, and let $F$ be a random variable. Then $F \alpha$ is integrable with respect to $B$ in the Russo-Vallois symmetric integral sense and $\int_{a}^{b} F \alpha_{w} d^{\circ} B_{w}=F \int_{a}^{b} \alpha_{w} d^{\circ} B_{w}$.

We are now ready to show the existence of delayed areas and volumes with respect to fBm.

5.2. Delayed Lévy areas. Before we turn to statements involving increments as functions of two parameters, let us deal first with fixed times $s, t$ :

Proposition 5.3. Let $B$ be a d-dimensional fractional Brownian motion, with Hurst parameter $H>1 / 4$. Then, for $s, t \in[0, T], v_{1} \in[-r, r], v_{2} \in[0, r]$, such that $v_{1}+v_{2} \geq 0$, the doubly delayed Lévy area, denoted by $\mathbf{B}_{s t}^{2}\left(v_{1}, v_{2}\right)$ and defined by (71), is well defined. In addition, we have $\mathbb{E}\left[\left|\mathbf{B}_{\text {st }}^{2}\left(v_{1}, v_{2}\right)\right|^{2}\right] \leq c|t-s|^{4 H}$ for a strictly positive constant $c=$ $c_{H, v_{1}, T}$ independent of $v_{2}$, exhibiting the following discontinuity phenomenon: we have $\lim _{v_{1} \rightarrow 0} c_{H, v_{1}, T}=\infty$, but $c_{H, 0, T}$ is finnite.

Remark 5.1. The discontinuity result on $c_{H, v_{1}, T}$ alluded to above is not a surprise, and had already been observed in [16].

Proof. As mentioned before, the case $H \geq 1 / 2$ is rather easy to handle, and we thus focus on $1 / 4<H<1 / 2$. It should also be mentioned that Lévy areas can be constructed in a similar way to [16], though an extra attention has to be paid in order to treat irregular cases, when $H$ approaches $1 / 4$. As a last preliminary remark, observe that, due to the stationarity property of the $\mathrm{fBm}$ we shall work without loss of generality on the interval $[0, t-s]$ instead of $\left[s-v_{2}, t-v_{2}\right]$ in the sequel, that is, $\mathbf{B}_{s t}^{\mathbf{2}}\left(v_{1}, v_{2}\right)$ behaves as $\mathbf{B}_{0, t-s}^{2}\left(v_{1}\right)=\mathbf{B}_{0, t-s}^{2}\left(v_{1}, 0\right)$.

1) Case $i=j$ and $v_{1} \geq 0$. Consider the process $\phi=\left(B_{-v_{1}}^{i}-B_{-v_{1}}^{i}\right) \mathbb{1}_{[0, t-s]}(\cdot)$. When $v_{1} \geq 0$, the arguments in [16, Proposition 5.2] for $1 / 3<H<1 / 2$ also hold for $1 / 4<H \leq 1 / 3$. Thus

$$
\left(\mathbf{B}_{0, t-s}^{2}\left(v_{1}\right)\right)^{i i}=I^{B^{i}}(\phi)+\operatorname{Tr}_{[0, t-s]} D^{B^{i}} \phi,
$$

where $I^{B^{i}}(\phi)$ denotes the divergence integral of $\phi$ with respect to $B^{i}$ and

$$
\operatorname{Tr}_{[0, t-s]} D^{B^{i}} \phi=\left\{\begin{array}{cl}
\frac{1}{2}(t-s)^{2 H}, & \text { if } v_{1}=0 \\
-H v_{1}^{2 H-1}(t-s)+\frac{1}{2}\left(\left(t-s+v_{1}\right)^{2 H}-v_{1}^{2 H}\right), & \text { if } v_{1}>0 .
\end{array}\right.
$$

In addition, one can also prove, as in [16], that

$$
\mathbb{E}\left|\left(\mathbf{B}_{0, t-s}^{2}\left(v_{1}\right)\right)^{i i}\right|^{2} \leq c_{H, v_{1}}|t-s|^{4 H},
$$


for any $v_{1} \in(0, r]$, where $\lim _{v_{1} \rightarrow 0} c_{H, v_{1}}=\infty$. On the other hand, the computations above also show that $\mathbb{E}\left|\left(\mathbf{B}_{0, t-s}^{2}\left(v_{1}, 0\right)\right)^{i i}\right|^{2} \leq c_{H, v_{1}}|t-s|^{4 H}$.

2) Case $i=j$ and $v_{1}<0$. When $v_{1}<0$, we will show that

$$
\left(\mathbf{B}_{0, t-s}^{2}\left(v_{1}\right)\right)^{i i}=I^{B^{i}}(\phi)+\operatorname{Tr}_{[0, t-s]} D^{B^{i}} \phi,
$$

where now

$$
\operatorname{Tr}_{[0, t-s]} D^{B^{i}} \phi=H\left(-v_{1}\right)^{2 H-1}(t-s)+\frac{1}{2}\left(\left|t-s+v_{1}\right|^{2 H}-\left(-v_{1}\right)^{2 H}\right) .
$$

Indeed, notice that $D_{r}^{B^{i}} \phi_{u}=\mathbb{1}_{\left[-v_{1}, u-v_{1}\right]}(r) \mathbb{1}_{[0, t-s]}(u)$ and furthermore, for $u \in[0, t-s]$ and $\varepsilon \in\left[0,-v_{1}\right]$, one can write

$$
\begin{aligned}
& \left\langle\mathbb{1}_{\left[-v_{1}, u-v_{1}\right]}, \mathbb{1}_{[u-\varepsilon, u+\varepsilon]}\right\rangle_{\mathcal{H}} \\
& =\frac{1}{2}\left(\left|-v_{1}+\varepsilon\right|^{2 H}-\left|-v_{1}-\varepsilon\right|^{2 H}+\left|-v_{1}-u-\varepsilon\right|^{2 H}-\left|-v_{1}-u+\varepsilon\right|^{2 H}\right) \\
& =\frac{1}{2}\left(\left(-v_{1}+\varepsilon\right)^{2 H}-\left(-v_{1}-\varepsilon\right)^{2 H}+\left|-v_{1}-u-\varepsilon\right|^{2 H}-\left|-v_{1}-u+\varepsilon\right|^{2 H}\right) .
\end{aligned}
$$

Performing now a Taylor expansion in a neighbourhood of $\varepsilon=0$, we get

$$
\left(-v_{1}+\varepsilon\right)^{2 H}-\left(-v_{1}-\varepsilon\right)^{2 H}=4 H\left(-v_{1}\right)^{2 H-1} \varepsilon+o\left(\varepsilon^{2}\right) .
$$

Thus, applying the dominated convergence theorem (details are left to the reader) we obtain

$$
\lim _{\varepsilon \rightarrow 0} \int_{0}^{t-s} \frac{1}{4 \varepsilon}\left(\left(-v_{1}+\varepsilon\right)^{2 H}-\left(-v_{1}-\varepsilon\right)^{2 H}\right) d u=H\left(-v_{1}\right)^{2 H-1}(t-s) .
$$

Along the same lines, by separating the cases $-v_{1} \geq t-s, 0<u<-v_{1}<t-s$ and $-v_{1} \leq u<t-s$, it can also be proved that

$$
\lim _{\varepsilon \rightarrow 0} \int_{0}^{t-s} \frac{1}{4 \varepsilon}\left(\left|-v_{1}-u-\varepsilon\right|^{2 H}-\left|-v_{1}-u+\varepsilon\right|^{2 H}\right) d u=\frac{1}{2}\left(\left|t-s+v_{1}\right|^{2 H}-\left(-v_{1}\right)^{2 H}\right) .
$$

We now obtain (73) by putting together (74) and (75).

Let us bound now $\operatorname{Tr}_{[0, t-s]} D^{B^{i}} \phi$ from expression (73): in the case $-v_{1} \geq t-s$, invoking the fact that, for $0<p<1$ and $a \geq b>0$, the inequality $a^{p}-b^{p} \leq(a-b)^{p}$ holds true, we obtain

$$
\begin{aligned}
\left|\operatorname{Tr}_{[0, t-s]} D^{B^{i}} \phi\right| & =H\left(-v_{1}\right)^{2 H-1}(t-s)+\frac{1}{2}\left(\left(-v_{1}\right)^{2 H}-\left(-v_{1}-(t-s)\right)^{2 H}\right) \\
& \leq H(t-s)^{2 H}+\frac{1}{2}\left(\left(-v_{1}\right)^{2 H}-\left(\left(-v_{1}\right)^{2 H}-(t-s)^{2 H}\right)\right) \leq(t-s)^{2 H}
\end{aligned}
$$

and in the case $-v_{1}<t-s$, we also have

$$
\begin{aligned}
\left|\operatorname{Tr}_{[0, t-s]} D^{B^{i}} \phi\right| & =H\left(-v_{1}\right)^{2 H-1}(t-s)+\frac{1}{2}\left|\left(t-s+v_{1}\right)^{2 H}-\left(-v_{1}\right)^{2 H}\right| \\
& \leq H\left(-v_{1}\right)^{2 H-1} T^{1-2 H}(t-s)^{2 H}+\frac{1}{2}\left((t-s)^{2 H}+\left(-v_{1}\right)^{2 H}+\left(-v_{1}\right)^{2 H}\right) \\
& \leq\left(H\left(-v_{1}\right)^{2 H-1} T^{1-2 H}+\frac{3}{2}\right)(t-s)^{2 H} .
\end{aligned}
$$

Thus, we have found

$$
\left|\operatorname{Tr}_{[0, t-s]} D^{B^{i}} \phi\right| \leq\left(H\left(-v_{1}\right)^{2 H-1} T^{1-2 H}+\frac{3}{2}\right)(t-s)^{2 H}, \quad \text { for all } \quad v_{1} \in[-r, 0) .
$$


We proceed now to bound the term $I^{B^{i}}(\phi)$ in (72): owing to (70), we have

$$
\begin{aligned}
D_{r}^{B^{i} I^{B^{i}}(\phi)} & =\left(B_{r-v_{1}}^{i}-B_{-v_{1}}^{i}\right) \mathbb{1}_{[0, t-s]}(r)+I^{B^{i}}\left(\mathbb{1}_{\left[-v_{1},-v_{1}\right]} \mathbb{1}_{[0, t-s]}(\cdot)\right) \\
& =\left(B_{r-v_{1}}^{i}-B_{-v_{1}}^{i}\right) \mathbb{1}_{[0, t-s]}(r)+I^{B^{i}}\left(\mathbb{1}_{\left[v_{1}+r, t-s\right]}(\cdot)\right) \mathbb{1}_{\left[-v_{1}, t-s-v_{1}\right]}(r) \\
& =\left(B_{r-v_{1}}^{i}-B_{-v_{1}}^{i}\right) \mathbb{1}_{[0, t-s]}(r)+\left(B_{t-s}^{i}-B_{v_{1}+r}^{i}\right) \mathbb{1}_{\left[-v_{1}, t-s-v_{1}\right]}(r) .
\end{aligned}
$$

Hence, thanks to (77) and using the same arguments as in the proof of [16, Propositon 5.2 ], we obtain

$$
\mathbb{E}\left|I^{B^{i}}(\phi)\right|^{2} \leq c_{H}|t-s|^{4 H}
$$

with a constant $c_{H}>0$ independent of $v_{1}$.

Finally, (76) and (78) imply $\mathbb{E}\left|\left(\mathbf{B}_{0, t-s}^{2}\left(v_{1}\right)\right)^{i i}\right|^{2} \leq c_{H, v_{1}}|t-s|^{4 H}$ for any $v_{1} \in[-r, 0)$, and thus, according to our stationarity argument:

$$
\mathbb{E}\left|\left(\mathbf{B}_{s t}^{2}\left(v_{1}, v_{2}\right)\right)^{i i}\right|^{2} \leq c_{H, v_{1}}|t-s|^{4 H},
$$

for any $v_{1} \in[-r, 0)$ and $v_{2} \in[0, r]$.

3) Case $i \neq j$. This case can be treated similarly to [16, Proposition 5.2], and yields the same kind of inequality as in equation (79).

Our claim $\mathbb{E}\left[\left|\mathbf{B}_{s t}^{2}\left(v_{1}, v_{2}\right)\right|^{2}\right] \leq c|t-s|^{4 H}$ now stems easily from the inequalities we have obtained for the 3 cases $i=j$ and $v_{1} \geq 0, i=j$ and $v_{1}<0$, and $i \neq j$.

We can go one step further, and state a result concerning $\mathbf{B}^{\mathbf{2}}$ as an increment.

Proposition 5.4. Let $\mathbf{B}^{2}$ be the increment defined at Proposition 5.9. Then $\mathbf{B}^{2}$ satisfies Hypothesis 4.1 and 4.9 .

Proof. First, we have to ensure the almost sure existence of $\mathbf{B}_{s t}^{2}\left(v_{1}, v_{2}\right)$ for all $s, t \in[0, T]$. This can be done by noticing that $\mathbf{B}_{s t}^{2}\left(v_{1}, v_{2}\right)$ is a random variable in the second chaos of the fractional Brownian motion $B$, on which all $L^{p}$-norms are equivalent for $p>1$. Hence we can write:

$$
\mathbb{E}\left|\left(\mathbf{B}_{s t}^{\mathbf{2}}\left(v_{1}, v_{2}\right)\right)^{i j}\right|^{p} \leq c_{H, v_{1}, p}|t-s|^{2 p H},
$$

for any $i, j \in\{1, \ldots, d\}$ and $p \geq 2$. With the same kind of calculations, one can also obtain the inequality

$$
\mathbb{E}\left|\left(\mathbf{B}_{s_{2} t_{2}}^{2}\left(v_{1}, v_{2}\right)\right)^{i j}-\left(\mathbf{B}_{s_{1} t_{1}}^{2}\left(v_{1}, v_{2}\right)\right)^{i j}\right|^{p} \leq c_{H, v_{1}, p}\left(\left|t_{2}-t_{1}\right|^{p H}+\left|s_{2}-s_{1}\right|^{p H}\right) .
$$

Then, a standard application of Kolmogorov's criterion yields the almost sure definition of the whole family $\left\{\mathbf{B}_{s t}^{2}\left(v_{1}, v_{2}\right) ; s, t \in[0, T]\right\}$, and its continuity as a function of $s$ and $t$.

Moreover, a direct application of Lemma 5.2 gives

$$
\delta \mathbf{B}^{2}\left(v_{1}, v_{2}\right)=\delta\left(B\left(v_{2}+v_{1}\right)\right) \otimes \delta\left(B\left(v_{2}\right)\right),
$$

and Fubini's theorem for Stratonovich integrals with respect to $B$ also yield easily Hypothesis 4.2. Finally, it is readily checked that $\mathbf{B}^{2}\left(v_{1}, v_{2}\right) \in \mathcal{C}_{2}^{2 \gamma}\left(\mathbb{R}^{d, d}\right)$ for any $1 / 4<\gamma<H$, $v_{1} \in[-r, r]$ (separating the case $v_{1}=0$ ) and $v_{2} \in[0, r]$. Indeed, it is sufficient to apply Corollary 4 in 9] (see also inequality (90) in [16]), having in mind the bound (80) and expression (81). 
5.3. Delayed volumes. We study now the term $\mathbf{B}^{\mathbf{3}}\left(v_{1}, v_{2}\right)$, starting from a similar statement as in Proposition 5.3:

Proposition 5.5. Let $B$ be a d-dimensional fractional Brownian motion, with Hurst parameter $H>1 / 4$. Then, for $s, t \in[0, T], v_{1} \in[-r, r], v_{2} \in[0, r]$, such that $v_{1}+v_{2} \geq 0$, the doubly delayed volume, denoted by $\mathbf{B}^{\mathbf{3}}\left(v_{1}, v_{2}\right)$ and defined by (71), is well defined. In addition, we have $\mathbb{E}\left[\left|\mathbf{B}^{\mathbf{3}}\left(v_{1}, v_{2}\right)\right|^{2}\right] \leq c|t-s|^{6 H}$ for a strictly positive constant $c=c_{H, T, v_{1}, v_{2}}$ such that it goes to $\infty$ if $v_{1} \rightarrow 0$ or $v_{2} \rightarrow 0$, but is also well defined if $v_{1}=v_{2}=0$.

Proof. Here again, we focus on the case $1 / 4<H<1 / 2$, and due tho the stationarity property of the $\mathrm{fBm}$, we shall work without loss of generality on the interval $[0, t-s]$ instead of $[s, t]$ in the sequel. For notational sake, we will also set $\tau=t-s$ in the remainder of the proof.

1) Case $i=j=k$. Consider the process $\psi=\left(\mathbf{B}_{0, \cdot}^{2}\left(v_{1}, v_{2}\right)^{i i}\right) \mathbb{1}_{[0, \tau]}(\cdot)$. We will define $\left(\mathbf{B}^{3}\left(v_{1}, v_{2}\right)\right)^{i i i}$ as $\int_{0}^{\tau} \psi_{u} d^{\circ} B_{u}^{i}$, which amounts to show that $\psi \in \mathbb{D}^{1,2}\left(\mathcal{H}_{K}([0, T])\right)$ and to compute the trace of the process $\psi$.

With this aim in mind, let us first compute the Malliavin derivative of $\psi$ : it is easily seen that

$$
\begin{aligned}
& D_{r}^{B^{i}} \psi_{u} \\
& =\left(B_{r-v_{1}}^{i}-B_{-v_{2}-v_{1}}^{i}\right) \mathbb{1}_{\left[-v_{2}, u-v_{2}\right]}(r) \mathbb{1}_{[0, \tau]}(u)+I^{B^{i}}\left(\mathbb{1}_{\left[-v_{2}-v_{1},-v_{1}\right]}(r) \mathbb{1}_{\left[-v_{2}, u-v_{2}\right]}(\cdot)\right) \mathbb{1}_{[0, \tau]}(u) \\
& =\left(B_{r-v_{1}}^{i}-B_{-v_{2}-v_{1}}^{i}\right) \mathbb{1}_{\left[-v_{2}, u-v_{2}\right]}(r) \mathbb{1}_{[0, \tau]}(u)+\left(B_{u-v_{2}}^{i}-B_{r+v_{1}}^{i}\right) \mathbb{1}_{\left[-v_{2}-v_{1}, u-v_{2}-v_{1}\right]}(r) \mathbb{1}_{[0, \tau]}(u) .
\end{aligned}
$$

From this identity, one can check that $\psi \in \mathbb{D}^{1,2}\left(\mathcal{H}_{K}([0, T])\right)$. We will now evaluate $\int_{0}^{\tau} \psi_{u} d^{\circ} B_{u}^{i}$ by separating the Skorokhod and the trace term in the symmetric integral.

(i) Evaluation of the trace term. We start by observing that $D^{B^{i}} \psi_{u}$ can also be written as:

$$
\begin{aligned}
D_{r}^{B^{i}} \psi_{u}= & I^{B^{i}}\left(\mathbb{1}_{\left[\cdot+v_{1}, u-v_{2}\right]}(r) \mathbb{1}_{\left[-v_{2}-v_{1}, u-v_{2}-v_{1}\right]}(\cdot)\right) \mathbb{1}_{[0, \tau]}(u) \\
& +I^{B^{i}}\left(\mathbb{1}_{\left[-v_{2}-v_{1},-v_{1}\right]}(r) \mathbb{1}_{\left[-v_{2}, u-v_{2}\right]}(\cdot)\right) \mathbb{1}_{[0, \tau]}(u)
\end{aligned}
$$

Apply then Fubini's Theorem in order to get

$$
\begin{aligned}
& \int_{0}^{\tau}\left\langle D^{B^{i}} \psi_{u}, \mathbb{1}_{[u-\varepsilon, u+\varepsilon]}\right\rangle_{\mathcal{H}} d u \\
& =\int_{-v_{2}-v_{1}}^{\tau-v_{2}-v_{1}}\left(\int_{w+v_{2}+v_{1}}^{\tau}\left\langle\mathbb{1}_{\left[w+v_{1}, u-v_{2}\right]}, \mathbb{1}_{[u-\varepsilon, u+\varepsilon]}\right\rangle_{\mathcal{H}} d u\right) d B_{w}^{i} \\
& \quad+\int_{-v_{2}}^{\tau-v_{2}}\left(\int_{w+v_{2}}^{\tau}\left\langle\mathbb{1}_{\left[-v_{2}-v_{1}, w-v_{1}\right]}, \mathbb{1}_{[u-\varepsilon, u+\varepsilon]}\right\rangle_{\mathcal{H}} d u\right) d B_{w}^{i}
\end{aligned}
$$

where the last two integrals have to be interpreted in the Wiener sense, and are welldefined according to the criterions in [18]. 
Let us evaluate the scalar product in (84): for a fixed $v_{2}>0, u \in\left[w+v_{2}+v_{1}, \tau\right]$, $w \in\left[-v_{2}-v_{1}, \tau-v_{2}-v_{1}\right]$ and $\varepsilon \in\left[0, v_{2}\right]$, we can write

$$
\begin{aligned}
& \left\langle\mathbb{1}_{\left[w+v_{1}, u-v_{2}\right]}, \mathbb{1}_{[u-\varepsilon, u+\varepsilon]}\right\rangle_{\mathcal{H}} \\
& =\frac{1}{2}\left(\left|-v_{2}+\varepsilon\right|^{2 H}-\left|-v_{2}-\varepsilon\right|^{2 H}+\left|w+v_{1}-u-\varepsilon\right|^{2 H}-\left|w+v_{1}-u+\varepsilon\right|^{2 H}\right) \\
& =\frac{1}{2}\left(\left(v_{2}-\varepsilon\right)^{2 H}-\left(v_{2}+\varepsilon\right)^{2 H}+\left(u-w-v_{1}+\varepsilon\right)^{2 H}-\left(u-w-v_{1}-\varepsilon\right)^{2 H}\right) \\
& =2 H\left(-v_{2}^{2 H-1}+\left(u-w-v_{1}\right)^{2 H-1}\right) \varepsilon+o\left(\varepsilon^{2}\right) .
\end{aligned}
$$

If $v_{2}=0$, one can prove similarly that for $\varepsilon$ small enough,

$$
\left\langle\mathbb{1}_{\left[w+v_{1}, u-v_{2}\right]}, \mathbb{1}_{[u-\varepsilon, u+\varepsilon]}\right\rangle_{\mathcal{H}}=2 H\left(u-w-v_{1}\right)^{2 H-1} \varepsilon+o\left(\varepsilon^{2}\right) .
$$

This yields easily the relation

$$
\lim _{\varepsilon \rightarrow 0} \frac{1}{2 \varepsilon}\left\langle\mathbb{1}_{\left[w+v_{1}, u-v_{2}\right]}, \mathbb{1}_{[u-\varepsilon, u+\varepsilon]}\right\rangle_{\mathcal{H}}=\left\{\begin{array}{cl}
H\left(-v_{2}^{2 H-1}+\left(u-w-v_{1}\right)^{2 H-1}\right) & \text { if } v_{2}>0 \\
H\left(u-w-v_{1}\right)^{2 H-1} & \text { if } v_{2}=0 .
\end{array}\right.
$$

The same kind of elementary arguments work for the scalar product in expression (85), and one obtains:

$$
\lim _{\varepsilon \rightarrow 0} \frac{1}{2 \varepsilon}\left\langle\mathbb{1}_{\left[-v_{2}-v_{1}, w-v_{1}\right]}, \mathbb{1}_{[u-\varepsilon, u+\varepsilon]}\right\rangle_{\mathcal{H}}=H\left(-\left(u-w+v_{1}\right)^{2 H-1}+\left(v_{2}+v_{1}+u\right)^{2 H-1}\right) .
$$

Thus, by an application of the dominated convergence theorem (whose details are left to the reader) we get, for a fixed $v_{2}>0$,

$$
\begin{aligned}
& \operatorname{Tr}_{[0, \tau]} D^{B^{i}} \psi \\
& =\int_{-v_{2}-v_{1}}^{\tau-v_{2}-v_{1}}\left(-H v_{2}^{2 H-1}\left(\tau-w-v_{2}-v_{1}\right)+\frac{1}{2}\left[\left(\tau-w-v_{1}\right)^{2 H}-v_{2}^{2 H}\right]\right) d B_{w}^{i} \\
& +\frac{1}{2} \int_{-v_{2}}^{\tau-v_{2}}\left(\left(v_{2}+v_{1}\right)^{2 H}-\left(\tau-w+v_{1}\right)^{2 H}+\left(\tau+v_{2}+v_{1}\right)^{2 H}-\left(2 v_{2}+v_{1}+w\right)^{2 H}\right) d B_{w}^{i}
\end{aligned}
$$

and for $v_{2}=0$, we end up with:

$$
\begin{aligned}
\operatorname{Tr}_{[0, \tau]} D^{B^{i}} \psi=\frac{1}{2} \int_{-v_{1}}^{\tau-v_{1}} & \left(\tau-w-v_{1}\right)^{2 H} d B_{w}^{i} \\
+ & \frac{1}{2} \int_{0}^{\tau}\left(v_{1}^{2 H}-\left(\tau-w+v_{1}\right)^{2 H}+\left(\tau+v_{1}\right)^{2 H}-\left(v_{1}+w\right)^{2 H}\right) d B_{w}^{i} .
\end{aligned}
$$

For the remainder of the paper, the relation $a \lesssim b$ stands for $a \leq C b$ with a universal constant $C$. Starting from equation (86), let us evaluate $\operatorname{Tr}_{[0, \tau]} D^{B^{i}} \psi$ for $v_{2}>0$. Observe first that one can write $\mathbb{E}\left[\left|\operatorname{Tr}_{[0, \tau]} D^{B^{i}} \psi\right|^{2}\right] \lesssim \sum_{l=1}^{4} J_{l}$, where $J_{l}$ can be decomposed itself as $J_{l}=\mathbb{E}\left[\left|\int_{0}^{\tau} F_{l}(w) d B_{w}^{i}\right|^{2}\right]$, with

$$
\begin{aligned}
& F_{1}(w)=(\tau-w), \quad F_{2}(w)=\left(\tau+v_{2}+v_{1}\right)^{2 H}-\left(v_{2}+v_{1}+w\right)^{2 H} \\
& F_{3}(w)=\left(v_{2}+v_{1}\right)^{2 H}-\left(\tau-w+v_{2}+v_{1}\right)^{2 H}, \quad F_{4}(w)=\left(\tau-w+v_{2}\right)^{2 H}-v_{2}^{2 H} .
\end{aligned}
$$

Thus, thanks to relation (67), we obtain:

$$
J_{l}=\left\|F_{l}\right\|_{\mathcal{H}([0, \tau])}^{2}=c_{H}\left\|\mathcal{D}_{-\tau}^{1 / 2-H} F_{l}\right\|_{L^{2}([0, \tau])}^{2}
$$


Furthermore, each $F_{l}$ is a power function, whose fractional derivative $\mathcal{D}_{-\tau}^{1 / 2-H} F_{l}$ can be computed explicitly. It is then easily shown that $\mathbb{E}\left|\operatorname{Tr}_{[0, \tau]} D^{B^{i}} \psi\right|^{2} \leq c_{H, v_{2}, T} \tau^{6 H}$, where $c_{H, v_{2}, T}=c_{H, T} v_{2}^{2(2 H-1)}+c_{H}$. Analogously, for $v_{2}=0$, we get $\mathbb{E}\left|\operatorname{Tr}_{[0, \tau]} D^{B^{i}} \psi\right|^{2} \leq c_{H} \tau^{6 H}$.

(ii) Evaluation of the Skorokhod term. We shall prove that $\mathbb{E}\left|I^{B^{i}}(\psi)\right|^{2} \leq c_{H, v_{1}, T} \tau^{6 H}$ and to this aim, let us decompose $\psi$ into its Skorokhod and trace part. This gives $\mathbb{E}\left|I^{B^{i}}(\psi)\right|^{2} \leq 2 \mathbb{E}\left|I^{B^{i}}\left(\psi_{1}\right)\right|^{2}+2 \mathbb{E}\left|I^{B^{i}}\left(\psi_{2}\right)\right|^{2}$, where

$$
\begin{aligned}
& \psi_{1}(w)=\int_{-v_{2}}^{w-v_{2}}\left[B_{u-v_{1}}^{i}-B_{-v_{2}-v_{1}}^{i}\right] d B_{u}^{i} \\
& \psi_{2}(w)=\operatorname{Tr}_{[0, w]} D^{B^{i}} \phi, \quad \text { with } \phi=\left(B_{\cdot-v_{1}}^{i}-B_{-v_{2}-v_{1}}^{i}\right) \mathbb{1}_{\left[-v_{2}, w-v_{2}\right]}(\cdot) .
\end{aligned}
$$

The proof that

$$
\mathbb{E}\left|I^{B^{i}}\left(\psi_{2}\right)\right|^{2} \leq c_{H, v_{1}, T} \tau^{6 H},
$$

where $c_{H, v_{1}, T} \rightarrow \infty$ if $v_{1} \rightarrow 0$ but is also well defined if $v_{1}=0$, can be obtained using the same arguments as for Step (i), and we then concentrate on the Skorokhod term $I^{B^{i}}\left(\psi_{1}\right)$.

To estimate $\mathbb{E}\left|I^{B^{i}}\left(\psi_{1}\right)\right|^{2}$, we use first identity (68), which can be read here as $\mathbb{E}\left|I^{B^{i}}\left(\psi_{1}\right)\right|^{2}$ $=\mathbb{E}\left[\left\langle\psi_{1}, D^{B^{i}} I^{B^{i}}\left(\psi_{1}\right)\right\rangle_{\mathcal{H}}\right]$. Taking into account relation (70), the expression (82) we have obtained for $D^{B^{i}} \psi_{1}$, and the isomorphism (67), we end up with

$$
\mathbb{E}\left[\left|I^{B^{i}}\left(\psi_{1}\right)\right|^{2}\right] \lesssim Q_{1}+Q_{2}+Q_{3}
$$

where $Q_{1}, Q_{2}, Q_{3}$ are respectively defined by:

$$
\begin{aligned}
Q_{1} & =\mathbb{E}\left\|\mathcal{D}_{-\tau}^{1 / 2-H} \psi_{1}\right\|_{L^{2}([0, \tau])}^{2} \\
Q_{2} & =\mathbb{E}\left\|\mathcal{D}_{-\left(\tau-v_{2}\right)}^{1 / 2-H}\left(\int_{+_{+v_{2}}^{\tau}}^{\tau}\left[B_{\cdot-v_{1}}^{i}-B_{-v_{2}-v_{1}}^{i}\right] d B_{w}^{i}\right)\right\|_{L^{2}\left(\left[-v_{2}, \tau-v_{2}\right]\right)}^{2} \\
Q_{3} & =\mathbb{E}\left\|\mathcal{D}_{-\left(\tau-v_{2}-v_{1}\right)}^{1 / 2-H}\left(\int_{+v_{2}+v_{1}}^{\tau}\left[B_{w-v_{2}}^{i}-B_{r+v_{1}}^{i}\right] d B_{w}^{i}\right)\right\|_{L^{2}\left(\left[-v_{2}-v_{1}, \tau-v_{2}-v_{1}\right]\right)}^{2} .
\end{aligned}
$$

We now estimate those 3 terms separately, starting with $Q_{1}$ : invoking the very definition (65) of the fractional derivative $\mathcal{D}_{-\tau}^{1 / 2-H}$, it is easily seen that $Q_{1} \lesssim A_{1}+A_{2}$, where

$$
\begin{aligned}
& A_{1}=\mathbb{E} \int_{0}^{\tau}\left(\int_{-v_{2}}^{r-v_{2}}\left[B_{u-v_{1}}^{i}-B_{-v_{2}-v_{1}}^{i}\right] d B_{u}^{i}\right)^{2} \frac{1}{(\tau-r)^{1-2 H}} d r \\
& A_{2}=\mathbb{E} \int_{0}^{\tau}\left(\int_{r}^{\tau} \frac{\int_{-v_{2}}^{w-v_{2}}\left[B_{u-v_{1}}^{i}-B_{-v_{2}-v_{1}}^{i}\right] d B_{u}^{i}-\int_{-v_{2}}^{r-v_{2}}\left[B_{u-v_{1}}^{i}-B_{-v_{2}-v_{1}}^{i}\right] d B_{u}^{i}}{(w-r)^{3 / 2-H}} d w\right)^{2} d r
\end{aligned}
$$

The term $A_{1}$ is easily bounded: according to Fubini's theorem and to our previous bounds on $\mathbf{B}^{2}$, we have

$$
\begin{aligned}
& A_{1}=\int_{0}^{\tau} \mathbb{E}\left(\int_{-v_{2}}^{r-v_{2}}\right. {\left.\left[B_{u-v_{1}}^{i}-B_{-v_{2}-v_{1}}^{i}\right] d B_{u}^{i}\right)^{2} \frac{1}{(\tau-r)^{1-2 H}} d r } \\
& \leq c_{H} \int_{0}^{\tau} r^{4 H} \frac{1}{(\tau-r)^{1-2 H}} d r \leq c_{H} \tau^{4 H} \int_{0}^{\tau} \frac{1}{(\tau-r)^{1-2 H}} d r=\frac{c_{H}}{2 H} \tau^{6 H} .
\end{aligned}
$$


The term $A_{2}$ is a little longer to treat. However, by resorting to the same kind of tools, one is able to prove that $A_{2} \leq c_{H} \tau^{6 H}$, and gathering the estimates on $A_{1}$ and $A_{2}$, we obtain $Q_{1} \leq c_{H} \tau^{6 H}$ as well. Finally, after some tedious computations which will be spared to the reader for sake of conciseness, we obtain the same kind of bound for $Q_{2}$ and $Q_{3}$.

Now one has to reverse our decomposition process: putting together our estimates on $Q_{1}, Q_{2}, Q_{3}$ and plugging them into (87), we get $\mathbb{E}\left[\left|I^{B^{i}}\left(\psi_{1}\right)\right|^{2}\right] \leq c_{H} \tau^{6 H}$, with a constant $c_{H}>0$ independent of $v_{1}, v_{2}$. Finally, gathering the bounds on the Skorokhod and the trace term, one obtains $\mathbb{E}\left[\left|\left(\mathbf{B}^{\mathbf{3}}\left(v_{1}, v_{2}\right)\right)^{i i i}\right|^{2}\right] \leq c|t-s|^{6 H}$.

2) Other cases. The previous arguments and computations can be simplified to obtain the desired result for the case $i=k \neq j$ and $j=k \neq i$. The cases $i=j \neq k$ and $i \neq j \neq k$ can be treated by means of Wiener integrals estimations. This finishes the proof of our claim $\mathbb{E}\left[\left|\mathbf{B}^{3}\left(v_{1}, v_{2}\right)\right|^{2}\right] \leq c|t-s|^{6 H}$.

As in the case of delayed Lévy areas, and with exactly the same kind of arguments, one can push forward the analysis in order to deal with $\mathbf{B}^{\mathbf{3}}$ as an increment:

Theorem 5.6. Let $\mathbf{B}^{\mathbf{3}}$ be the increment defined at Proposition 5.5. Then $\mathbf{B}^{\mathbf{3}}$ satisfies Hypothesis 4.1. Taking into account Proposition 5.4, Theorem 4.0 can thus be applied almost surely to the paths of the d-dimensional fBm with Hurst parameter $H>1 / 4$.

Acknowledgment: I. Torrecilla wishes to thank the IECN (Institut Élie Cartan Nancy) for its warm hospitality during a visit in 2008, which served to settle the basis of the current paper.

\section{REFERENCES}

[1] Alòs, E., Léon, J. L., Nualart., D.: Stratonovich calculus for fractional Brownian motion with Hurst parameter less than 1/2. Taiwanese J. Math. 5 (2001), 609-632.

[2] Cass, T., Friz, P: Densities for Rough Differential Equations under Hörmander's Condition. Preprint (2007).

[3] Cass, T., Friz, P., Victoir, N.: Non-degeneracy of Wiener functionals arising from rough differential equations. Preprint (2007).

[4] Coutin, L., Qian, Z.: Stochastic rough path analysis and fractional Brownian motion. Probab. Theory Related Fields 122 (2002), 108-140.

[5] Deya, A., Tindel, S.: Fractional Volterra equations 2: generalized convolutional integrals. Preprint (2008).

[6] Ferrante M., Rovira C.: Stochastic delay differential equations driven by fractional Brownian motion with Hurst parameter $H>1 / 2$. Bernoulli 12 (1) (2006), 85-100.

[7] Friz, P.: Continuity of the Itô-map for Hölder rough paths with applications to the support theorem in Hölder norm. Probability and partial differential equations in modern applied mathematics, 117-135, IMA Vol. Math. Appl., 140, Springer, New York, 2005.

[8] Friz, P., Victoir, N.: Multidimensional dimensional processes seen as rough paths. Cambridge University Press, to appear.

[9] Gubinelli, M.: Controlling Rough Paths. J. Funct. Anal. 216, (2004), 86-140.

[10] Gubinelli, M.: Ramification of rough paths. Preprint arXiv:math.CA/ 0610300 v1, (2006).

[11] Gubinelli, M., Tindel, S.: Rough evolution equations. Preprint (2008).

[12] Hoff, B.: The Brownian Frame Process as a Rough Path. Preprint (2006).

[13] Lyons, T.J., Qian, Z.: System control and rough paths. Oxford Mathematical Monographs. Oxford Science Publications. Oxford University Press, Oxford, (2002).

[14] Mohammed, S.-E. A.: Stochastic functional differential equations. Research Notes in Mathematics, 99. Boston-London-Melbourne: Pitman Advanced Publishing Program, (1984). 
[15] Neuenkirch, A., Nourdin, I., Rößler, A., Tindel, S.: Trees and asymptotic developments for fractional differential equations. Preprint arXiv:math.PR/0611306 v1, (2006). To appear in Ann. Inst. H. Poincaré, Probab. Stat.

[16] Neuenkirch, A., Nourdin, I., Tindel, S.: Delay Equations driven by Rough Paths. Elec. J. Probab. 13 (2008), 2031-2068.

[17] Nualart, D.: The Malliavin Calculus and Related Topics. Probability and its Applications. Springer-Verlag, 2nd Edition, (2006).

[18] Pipiras V., Taqqu, M. S.: Integration questions related to fractional Brownian motion. Probab. Theory Relat. Fields 118 (2), (2000), 251-291.

[19] Russo, F., Vallois, P.: Forward, backward and symmetric stochastic integration. Probab. Theory Relat. Fields 97 (1993), 403-421.

[20] Samko, S. G., Kilbas, A. A., Marichev, O. I.: Fractional Integrals and Derivatives. Gordon and Breach, New York. (1993).

[21] Samorodnitsky, G., Taqqu, M. S.: Stable non-Gaussian random processes. Chapman and Hall, (1994).

[22] Zähle, M.: Integration with respect to fractal functions and stochastic calculus I. Probab. Theory Relat. Fields 111, (1998), 333-374.

Samy Tindel: Institut Élie Cartan Nancy, B.P. 239, 54506 Vandouvre-lès-Nancy Cedex, France. Email:tindel@iecn.u-nancy.fr

Iván Torrecilla: Facultat de Matemàtiques, Universitat de Barcelona, Gran Via 585, 08007 Barcelona, Spain. Email: itorrecillatarantino@gmail.com. Supported by the grants HF2005-0038, MTM200601351 from the Dirección General de Investigación, Ministerio de Educación y Ciencia, Spain. 